\title{
A Review on Self-Recovery Regulation (SR) Technique for Unbalance Vibration of High-End Equipment
}

\author{
Xin $\operatorname{Pan}^{1,2^{*}} \mathbb{C}$, Jiaqiao $\mathrm{Lu}^{2}$, Jiaji Huo ${ }^{1}$, Jinji Gao ${ }^{1,2^{*}}$ and Haiqi Wu ${ }^{1}$
}

\begin{abstract}
The high-end equipment represented by high-end machine tools and aero-engines is the core component of the national intelligent manufacturing plan, and the mass unbalance is the main reason for its excessive vibration, that seriously impacts the operation efficiency and running life of the equipment. In order to change the traditional way that the fault of equipment can only be repaired by human, the self-recovery mechanism of human and animal are given to the equipment in this paper, which forms the self-recovery regulation (SR) system for unbalance vibration of high-end equipment. The system can online generate the self-recovery force to restrain the unbalance vibration of the equipment in operation, which is an important direction for the development of the equipment to the advanced intelligent stage. Based on the basic principles of SR technique, the typical engineering application cases of this technique in the field of aeroengine and high-end machine tools are introduced, and four related studies promoting the development of this technique are summarized and analyzed in turn. It includes feature extraction, imbalance location, regulation method and balancing actuator. Self-recovery Regulation (SR) Technique is an important way to realize intelligent manufacturing and intelligent maintenance. Relevant research can lay a technical foundation for the development of high-end equipment with self-health function.
\end{abstract}

Keywords: Unbalance vibration, Self-recovery regulation, Active balancing, High-end equipment, Self-health

\section{Introduction}

With the proposal and development of technologies, such as "Made in China 2025" and "Industry 4.0", highend equipment represented by high-end machine tools and aero-engines has become a key research field at home and abroad [1,2]. Excessive vibration caused by mass unbalance is a typical fault of high-end rotating equipment. For high-end machine tools, high-speed machining can improve machining efficiency several times or even dozens of times, and obtain better product processing accuracy and surface quality. However, mass unbalance restricts the increase of machine tool

\footnotetext{
*Correspondence: panxin@mail.buct.edu.cn; gaojinji@263.net

${ }^{1}$ Beijing Key Laboratory of Health Monitoring and Self-recovery for High-end Mechanical Equipment, Beijing University of Chemical Technology, Beijing 100029, China

Full list of author information is available at the end of the article
}

speed, even if there is a slight unbalance on the grinding wheel or cutting tool, a great unbalanced force will be generated, which lead to the aggravation of machine tool vibration and seriously affect the machining accuracy. For the aero-engine, several factors which include the structural deformation of the rotor system caused by the change of flight state, blade wear and impact, will lead to real-time changes in the unbalance state of the rotor system. The mass unbalance will cause engine vibration exceeding the standard and is easy to cause accidents including rotor rubbing, bearing damage and blade fracture [3-5]. Therefore, scholars at home and abroad have been committed to the research on the unbalance vibration of high-end equipment. In the past 5 years (2015-2019), a total of 2582 articles related to "unbalance vibration" and "mass imbalance" are searched by Web of Science database, of which $28.7 \%$ are in China and $21.3 \%$ in the United States. The 
academic journals with more related articles are as follows: Mechanical Systems and Signal Processing, Journal of Sound and Vibration, Journal of Vibration and Control, Mechanism and Machine Theory, and Journal of Vibration and Acoustics.

After diagnosis, location and warning for the fault of the equipment, it is usually necessary for the maintainer to repair the equipment after shutdown, which is not conducive to the improvement of equipment intelligence. The Institute for Strategic Research in Advanced Manufacturing of the Chinese Academy of Sciences points out that the intelligent level of equipment will be essentially improved by 2025, from controllability and automation to the advanced intelligent stage of self-maintenance and self-adaptation [6]. The demand of the project promotes the development of the technique related to online fault elimination. Fault self-recovery regulation (FSR) technique is that equipment are given the self-recovery function of people and animals which maintain a healthy state spontaneously, so that the self-recovery force can be generated to restrain the possible damage force during the operation of equipment. Therefore, the equipment can eliminate faults or do not have fault conditions in operation. It is an effective scheme to ensure high-end equipment to maintain its own healthy state and longterm stable operation $[7,8]$. The consultation project of the Chinese Academy of Engineering (Proposal of equipment failure self-recovery engineering and its application in China), points out that the fault self-recovery principle and its engineering application in complex systems of equipment can effectively prevent and eliminate faults and reduce the dependence of equipment on people [9].

After applying the fault self-recovery principle to the field of unbalance vibration suppression, the self-recovery regulation (SR) technique for unbalance vibration has been formed, which can restrain the unbalance vibration of the rotor system in real time in the course of the operation of the equipment. Therefore, it is also known as "online automatic balancing technique" [10]. At present, this technique has been implemented inside the field of highend machine tools and aero-engines, and the application effect is remarkable. For instance, the automatic balancing technique has been applied to the turboprop engine of a certain type of transport aircraft in USA, which can reduce the vibration at the engine propeller by more than 90\%; the technology has been applied to the high-end grinding machine in many countries including USA, Germany and Italy, and the unbalance vibration amplitude of the grinding wheel can always be controlled below $0.4 \mu \mathrm{m}$ $[11,12]$. The related research in China started relatively late, and now it is mainly carried out by universities such as Xi'an Jiaotong University, Beijing University of Chemical Technology, Zhejiang University, and Harbin Institute of Technology. However, there have been no reports on practical engineering application [13-16].

The SR technique for unbalance vibration is mainly composed of data acquisition, feature extraction, imbalance location, regulation method and execution. Therefore, this paper intends to make an in-depth summary of the above technique. In Section 2, the basic principle of SR system is described, and the SR for unbalance vibration is analyzed. In Section 3, based on the practical application cases of online automatic balancing technique, the engineering significance of this technique is described. In the following 4 sections, the key research contents of the SR technique, including feature extraction, imbalance location, regulation method and balancing actuator, are summarized respectively.

\section{Basic Principles}

\subsection{SR System}

On the basis of studying the principle of FSR, the SR of vibration and noise is proposed referring to the methods of "molecular targeted therapy" in contemporary medical science, which endows the machine with the ability to maintain a healthy state spontaneously. It is a new direction of bionic self-recovery principle and FSR system of equipment [17].

The typical FSR system takes the complex system of high-end equipment as the research object, and aims at "fast, accurate, precise and stable". As shown in Figure 1, the system should be composed of three parts, including Data collector, Controller and Actuator. (1) Data collector is used to achieve the function of information acquisition, including monitoring of sensitive parameters, data

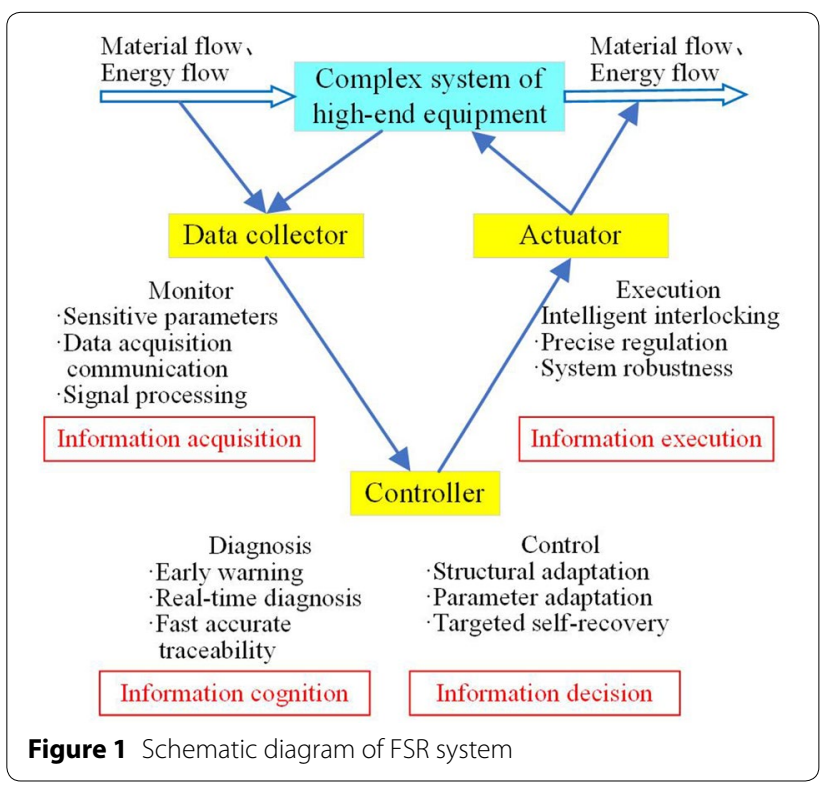


communication and signal pre-processing. (2) Controller is used to realize the functions of information cognition and information decision-making, including early warning of faults, real-time diagnosis, fast accurate traceability, parameter adaptation, and structural adaptation. (3) Actuator is used to realize the function of information execution, including intelligent interlocking, fine and stable regulation, etc. During the operation of the equipment, all parts of the SR system work together to quickly capture fault information, accurately locate the fault location and form control instructions, and generate a selfrecovery force by the robust action of the actuator. There are no conditions to form faults or the faults are eliminated in the bud by the regulation system. Therefore, the equipment can run without fault or can be restored from an abnormal state to a normal state without stopping, which ensures the long-term healthy operation of the equipment.

\subsection{SR for Unbalance Vibration}

The vibration differential equation of the rotor system may be shown as:

$$
M \ddot{x}+(C+\omega G) \dot{x}+K x=F_{0} \cos (\omega t+\varphi),
$$

where $M, C, G$ and $K$ are the matrix of the rotor, representing mass, damping, gyroscopic moment and stiffness, respectively. When the unbalance fault force on the rotor system is $F_{0}$, the differential equation can be solved, and the vibration amplitude caused by the unbalance fault is obtained as shown:

$$
x=\frac{F_{0}}{\sqrt{\left(K-M \omega^{2}\right)^{2}+(C+\omega G)^{2} \omega^{2}}} .
$$

In order to control the unbalance vibration, a balancing actuator needs to be pre-installed on the rotor and rotates synchronously with the rotor during a run. Assuming that the mass of the actuator is $m$ and the unbalance self-recovery force generated by the actuator is $F_{1}$, Eq. (1) may be revised as:

$$
(M+m) \ddot{x}+(C+\omega G) \dot{x}+K x=\left(F_{0}-F_{1}\right) \cos (\omega t+\varphi) .
$$

Therefore, when the controller of the SR system changes the internal mass distribution of the balancing actuator, the self-recovery force F1 generated by the balancing actuator gradually counteracts the unbalance failure force F0 of the system. When the two forces are approximately equal, the vibration of the rotor system can be decreased to the allowable range.

The principle block diagram of the SR system for unbalance vibration is shown in Figure 2. Taking the unbalance vibration of high-end equipment as the control target, the system can be divided into four layers from the view of information exchange. (1) Condition monitoring layer is used to analyze the data requirements of the SR system, select the appropriate sensors to measure the required physical quantities at the appropriate locations, and output the data according to the defined signal format. (2) Fault diagnosis layer is used to extract features of multi-dimensional signals, especially unbalance vibration components, through signal processing technique such as filtering, spectral analysis and principal component analysis, and online locate the unbalance vector when the unbalance vibration beyond the preset threshold. (3) Taking non-overshoot as the goal, target control layer is used to optimize the control path and output control commands, based on the running state, load conditions and historical data of the equipment. (4) According to the dynamic characteristics of the equipment, self-recovery execution layer is used to complete the design and layout of the balancing actuator, accurately execute the control commands of the controller, and online suppress the unbalance vibration of the equipment. Finally, the SR system for unbalance vibration of high-end equipment with four characteristics is formed, that is, state self-perception, real-time self-analysis, intelligent self-decision and precise self-execution.

\section{Application Cases}

The above-mentioned SR technique for unbalance vibration is mainly used in the fields of aero-engines and high-end machine tools at present. Two representative products applied to these two fields are introduced in detail as follows.

\subsection{Propeller Balancing System of Areo-engine}

For aero-engines, excessive unbalance vibration will significantly increase engine parts damage and fuel consumption, and reduce engine operation efficiency and service life. At present, there are three commonly used solutions [18]. (1) The engine is disassembled back to the factory, and the parts are reassembled after the dynamic balance is completed on the dynamic balancing machine [19-21]. (2) There is no need to disassemble the engine, the trial operation of the whole engine is accomplished on the engine test bench and the vibration parameters are measured, and the dynamic balance is carried out on the bench [18]. (3) The vibration parameters of the engine are monitored during the flight, and the unbalance vector calculation and balancing operation are carried out after the aircraft has landed, then the balancing process can be completed on the aircraft $[22,23]$. Among them, both solution 1 and 2 require a long maintenance cycle, which is time-consuming and laborious, and seriously affect the service 


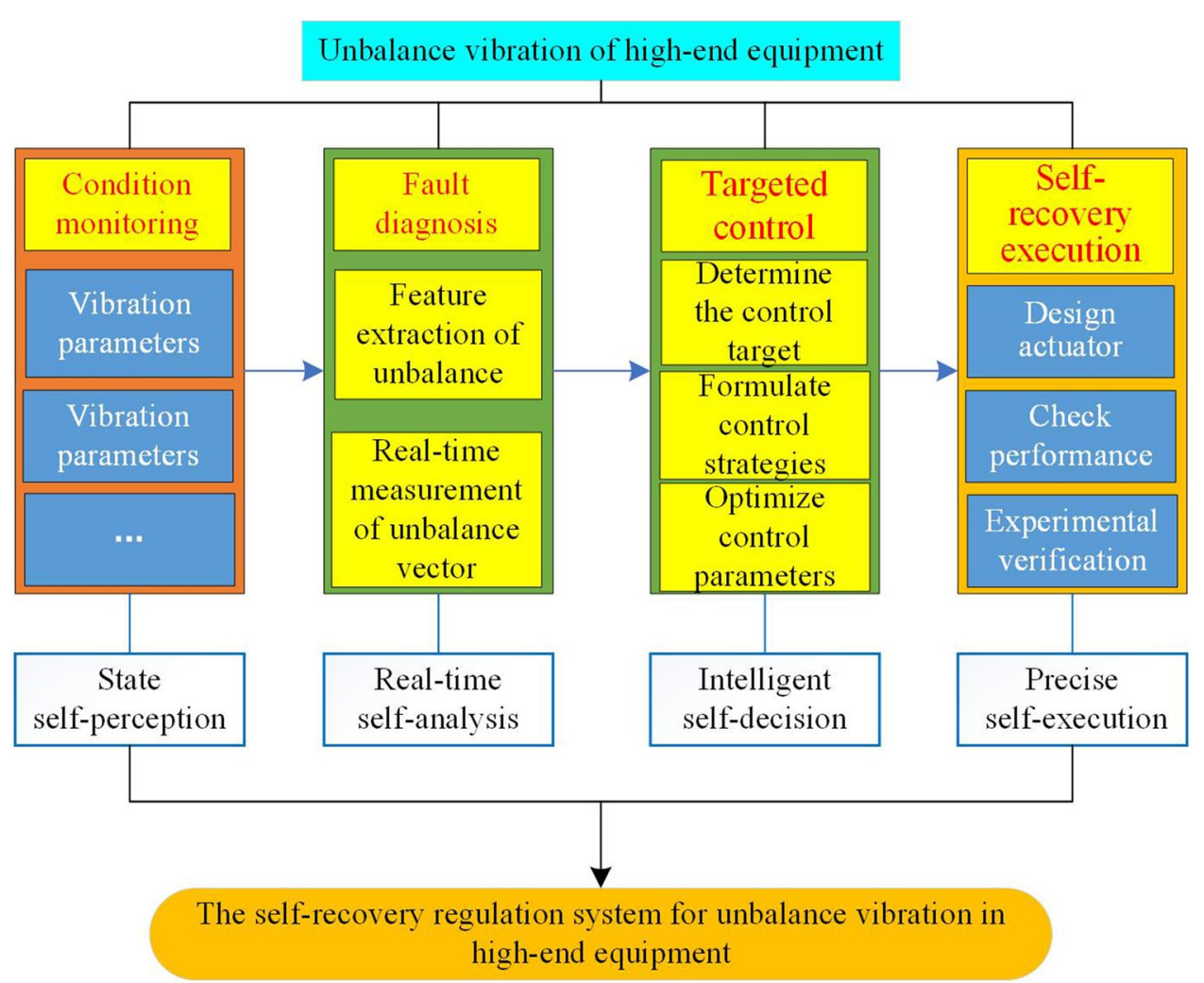

Figure 2 Principle block diagram of SR system for unbalance vibration

time of the engine. Although solution 3 has simplified the dynamic balance operation, there is still a difference between the balance condition and the actual operation condition of the aero-engine, hence the balance effect is limited.

Therefore, the in-flight propeller balancing system (IPBS) is developed to solve the above problems by a company of USA. The system can surveil the vibration parameters of the turboprop engine in real time while the aircraft is flying, and restrain the unbalance vibration of the propeller in real time under a variety of operating conditions, such as aircraft start-up, normal operation or parking. As shown in Figure 3, IPBS is mainly made up of three parts, that is, the sensor, the controller and the balancing actuator. The balancing actuator is pre-installed on the engine rotating shaft, and the vibration of the rotating shaft is monitored in real-time by the accelerometer. When the measured vibration value of the rotor exceeds the prescribed safety range, the system calculates the corresponding unbalance through the monitoring data of the sensor, then the controller transmits instructions to adjust the balancing actuator to automatically balance the rotor. In addition, the system can diagnose the engine through monitoring data, and the diagnostic

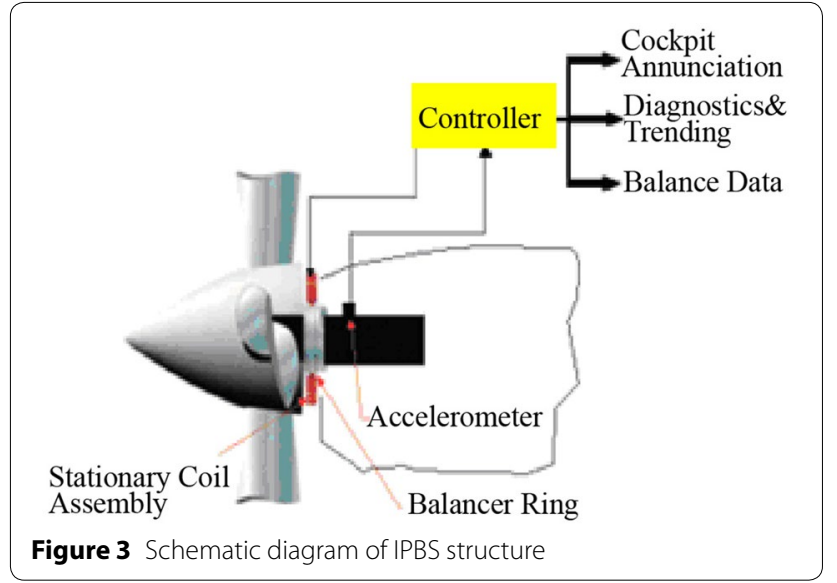

information can be used to forecast the health condition of the engine system and optimize maintenance [11, 24].

The system has been successfully applied to four turboprop engines of a large transport aircraft. As shown in Figure 4, each of the turboprop engines is equipped with a balancing actuator, the four balancing actuators share a controller, and the power supply of the balance system is provided by the cockpit. The installation of the 


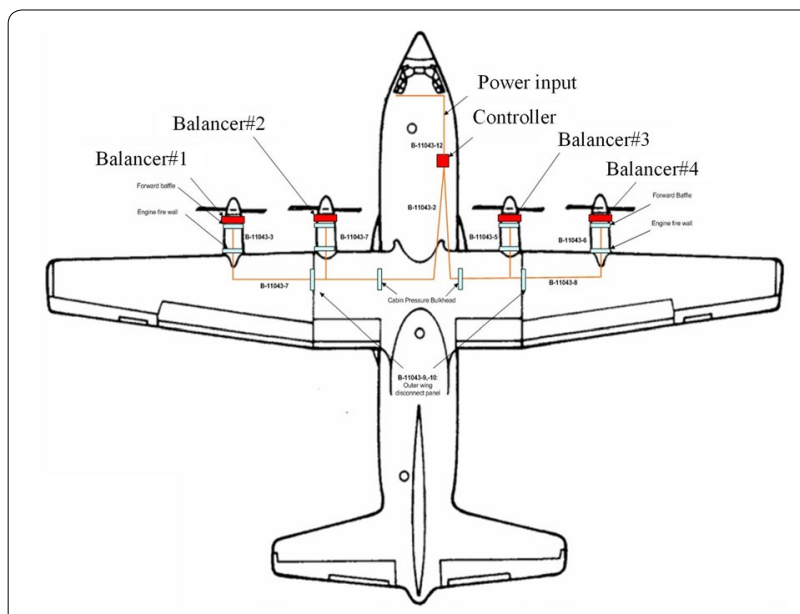

Figure 4 Installation diagram of the automatic balancing system

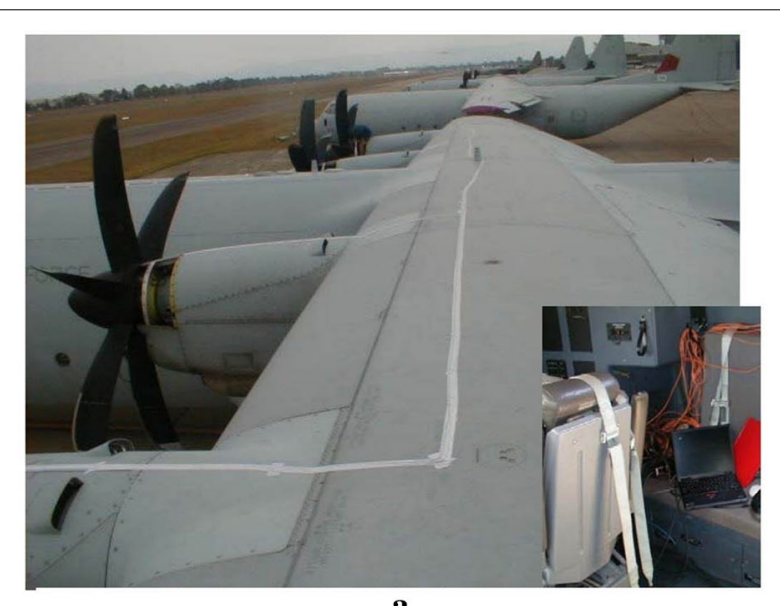

$\mathbf{a}$

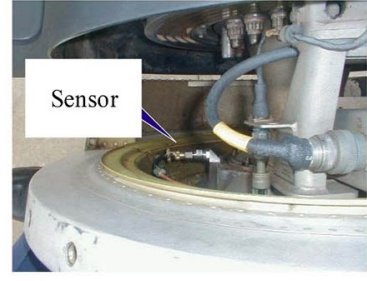

b

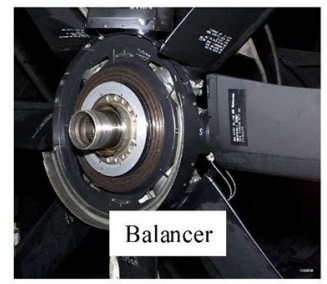

c
Figure $\mathbf{5}$ Physical diagram of propeller automatic balancing system: a related wiring, $\mathbf{b}$ vibration sensor, $\mathbf{c}$ balance actuator

vibration sensor and the actuator, and the wiring of the flight deck and cockpit, are shown in Figure 5, the performance parameters of the balancing actuator are shown in Table 1, and the vibration suppression effect of the system under several flight conditions is shown in Figure 6.

According to the actual test results, the system can restrain the unbalance vibration of turboprop engines in real time under all flight conditions, so that the vibration
Table 1 Performance parameters of the IPBS

\begin{tabular}{ll}
\hline Performance parameter & Value \\
\hline Rotation speed (r/min) & 1020 \\
Balancing capacity & $0.05-220 \mathrm{Oz}-\mathrm{in}$ \\
Balancing time $(\mathrm{s})$ & $3.6-15840 \mathrm{~g} \mathrm{~cm}$ \\
\hline
\end{tabular}

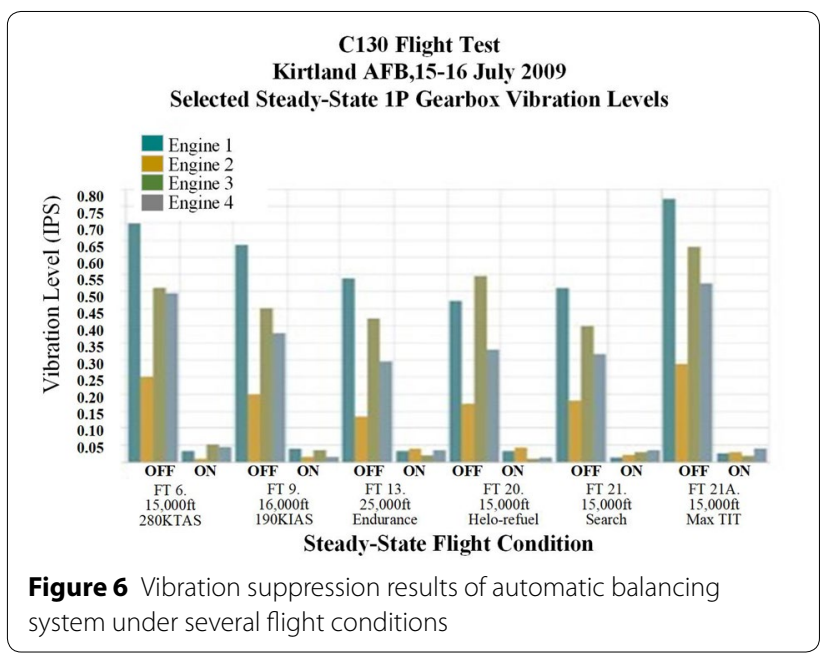

of the four engines can always be kept below $0.05 \mathrm{inch} / \mathrm{s}$, and the maximum vibration reduction can reach more than $90 \%$. At the same time, during the testing process of 250 flight-hour, the balancing system performed a total of 9000 dynamic balancing operations, that is, in order to ensure that the engine vibration is always below the safety setting value, the engine needs to be dynamically balanced once per $1.6 \mathrm{~min}$. The data further validates the necessity of installing the balancing system for aero-engine.

\subsection{Grinding Wheel Balancing System of Grinding-Machine} As the last process of machining, grinding have an important impact on the overall level of the machining industry. Due to the inevitable problem of uneven adsorption of grinding fluid or uneven wear of grinding wheel during the grinding process, even if the grinding wheel has been pre-balanced before installation, the balance state of grinding wheel will still be destroyed after a period of use, resulting in excessive vibration of the machine. If the parts continue to be processed, the surface quality of the subsequent parts cannot be guaranteed. While in the conventional dynamic balance process of grinding wheel, the grinding wheel often has to be disassembled and installed repeatedly, which is time-consuming and 
laborious, and it is not easy to ensure the machining consistency of batches of parts.

With the intention to resolve the above problems, domestic and foreign scholars have carried out continuous research on on-line automatic balancing technique of grinding wheels. Several world-leading manufacturing countries have formed the related products, such as USA, Germany and Italy.

As shown in Figure 7, the typical automatic balancing system for grinding wheel is mainly composed of vibration sensors, controllers and balancing actuators. Among them, the vibration sensor is a piezoelectric sensor, which is used to monitor the vibration of the grinding wheel. The controller diagnoses the vibration data monitored by the sensor, and displays the diagnosis results on the electronic screen, then sends control instructions to control the actuator. The actuator is installed on the side of the grinding wheel through the flange and rotates synchronously with the grinding wheel, and after receiving the balance instruction, the compensation quality is formed by changing its own mass distribution [12]. The performance parameters of commonly used balancing actuators are shown in Table 2. In actual applications, the actuator can be selected according to the dynamic balance requirements of the grinding wheel.

When the balancing system is installed on the grinding machine, the vibration level of the grinding wheel can always be controlled to less than $0.4 \mu \mathrm{m}$. Compared with $2.0 \mu \mathrm{m}$ of the traditional dynamic balance method of grinding wheel, the control effect is improved by more than 5 times. As shown in Figure 8, the mechanical wear of the grinder and the start and stop times of machining can be significantly reduced, and the linear velocity of the grinding wheel can be further increased, resulting in higher machining efficiency and product quality.

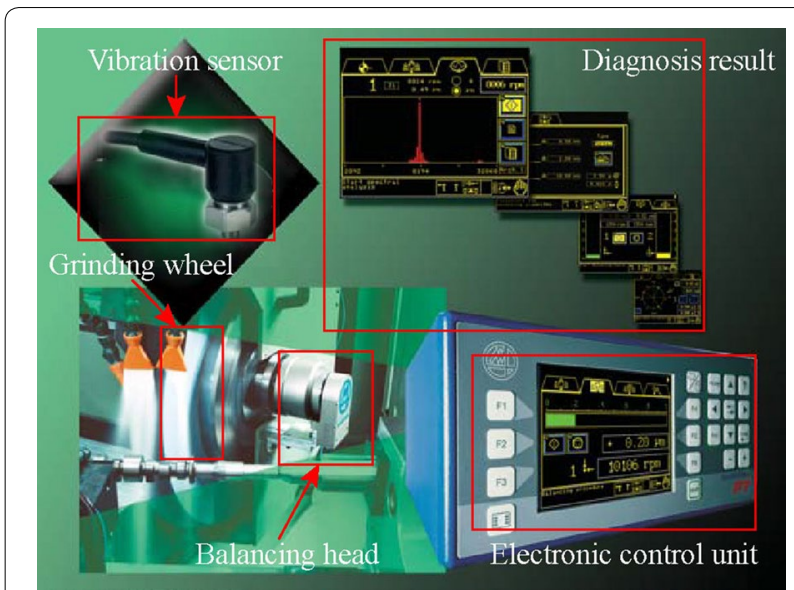

Figure 7 Diagram of automatic balancing system for grinding wheel

\section{Feature Extraction}

According to the correlation analysis of Section 2.2, it is known that the premise of SR is to complete feature extraction and determine whether the unbalance vibration exceeds the standard value. Therefore, the feature extraction and processing of vibration signals is the first step of SR. At present, the methods of feature extraction are generally classified into 3 categories: Time domain type; Frequency domain type; Joint time-frequency type [25-29]. At the same time, with the promotion of intelligent algorithms, feature extraction technology based on intelligent algorithms is gradually becoming a research hotspot.

\subsection{Time Domain Method}

The feature extraction based on the method is mainly statistical analysis. The time domain features of vibration signals based on statistical analysis can be divided into dimensional and dimensionless characteristic parameters. The dimensional characteristic parameters mainly include mean value, peak value, and root mean square of the vibration signal, while the dimensionless

\begin{tabular}{|c|c|c|c|}
\hline Model & $\begin{array}{l}\text { Balancing } \\
\text { capacity }(\mathrm{g} \cdot \mathrm{cm})\end{array}$ & Diameter $(\mathrm{mm})$ & $\begin{array}{l}\text { Rotation } \\
\text { speed ( } r / \\
\text { min) }\end{array}$ \\
\hline FT 900 & 900 & 112 & 4000 \\
\hline FT 1300 & 1300 & 112 & 4000 \\
\hline FT 2000 & 2000 & 112 & 3000 \\
\hline FT 3000 & 3000 & 132 & 3000 \\
\hline FT 4500 & 4500 & 132 & 2000 \\
\hline
\end{tabular}

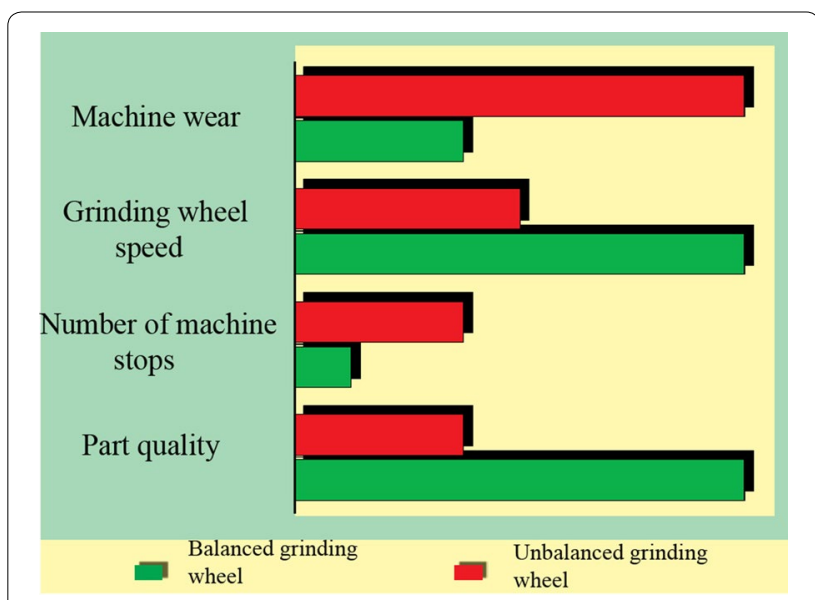

Figure 8 Comparison of the effect before and after dynamic balance of grinding wheel 
characteristic parameters are obtained by the combination of dimensional parameters, including kurtosis factor, kurtosis index, pulse index, and so on.

Deng et al. [30] proposed a complete fault characteristic extraction approach. One basis for this approach is local mean decomposition (LMD) which can be used to process the original vibration to generate the product functions (PFS), and the other is Teager energy kurtosis (TEK) which can be used to calculate at PFS. The corresponding TEK of each Teager energy data can be used to identify the failure by this method. The effectiveness of the method for rotor-bearing system was verified by experiments.

Imbalance and shaft bending are the two most common types of fault in rotary machinery, and the two types of faults display similar characteristics. In order to extract and identify the features of these two faults, Sanches et al. [31] put forward a time domain recognition method based on correlation analysis and model reduction. Since the minimum entropy deconvolution (MED) can not identify multiple faults of rotating machinery, He et al. [32] proposed a multi-fault detection technique which based on spectral kurtosis (SK) and MED. The layout of the vacuum pump test-bed is shown in Figure 9, and the flowchart of this technique is shown in Figure 10. In this technique, the signal is decomposed via SK, and each decomposed signal is refined by MED, so as to identify a variety of faults.

However, if the fault component contained in the signal is complex, it is difficult to make an accurate judgment of the fault only through the time domain eigenvalues, so the frequency domain analysis is accomplished for further analysis.

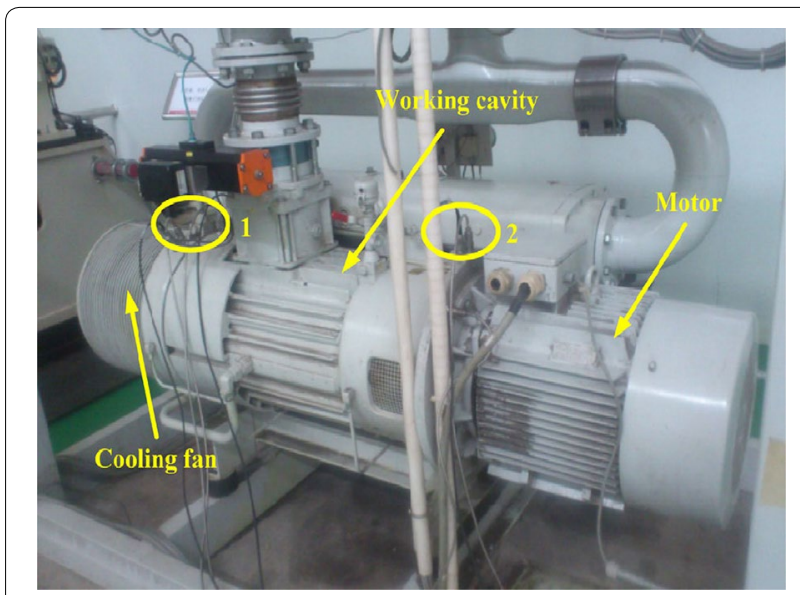

Figure 9 Vacuum pump test-bed

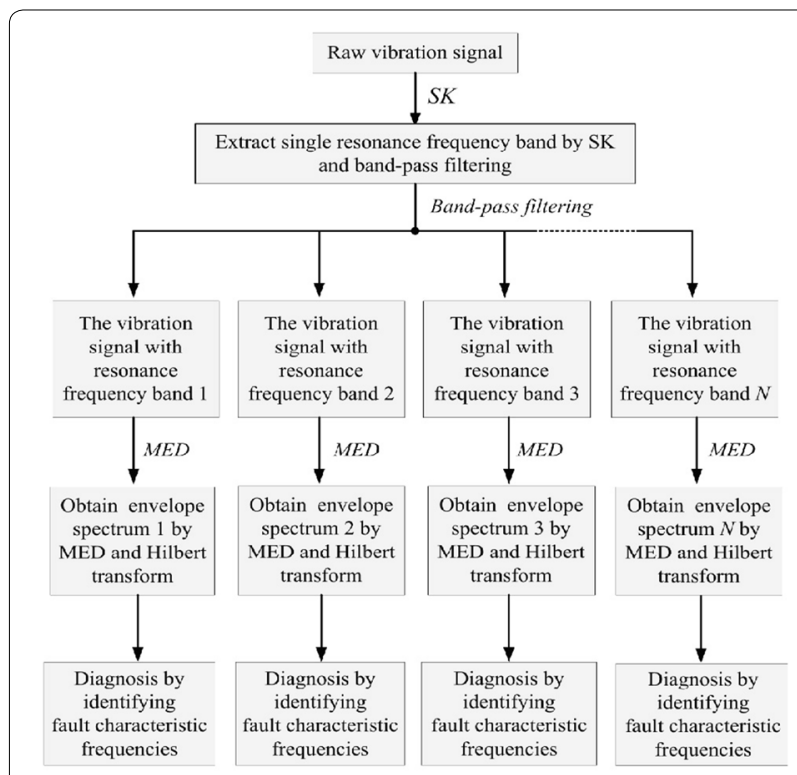

Figure 10 Technical process of He et al. [32]

\subsection{Frequency Domain Method}

This method refers to the processing of the time domain signal through Fourier transform, and the signal is reflected in the form of amplitude-frequency. Through the observation of the spectrum, the diagnosis of the data can be realized. The main feature extraction methods based on frequency domain are cepstrum analysis, power spectrum analysis and holo-spectrum analysis.

Cepstrum is an effective tool to detect periodic components in complex maps, and it has been broadly utilized for fault monitoring and diagnosis. For high-speed and large rotating machinery, its rotation condition is complex, especially when there are faults consisting of misalignment, gear defects, oil whirl, and so on, the vibration will become more complex. If there is a complex periodic structure on the spectrum, the recognition ability can be enhanced by analyzing it with cepstrum.

With a view to improving the production efficiency, product quality and reduce the processing costs of the workpiece, spindles and machine tools must work in the best environment with as little error as possible. The detection and diagnosis of spindle unbalance (UB), misaligned (MA), bent shaft (BS) or rolling bearing has always been considered as a difficult problem in rotary machining monitoring. David Ibarra-Zarate et al. [33] proposed a method based on cepstrum pre-whitening analysis. Figure 11 shows the signal extraction and selection process of this method.

Rotor unbalance often leads to rubbing in aeroengine, and it is very difficult to diagnose the friction position. Chen et al. $[34,35]$ used cepstrum to analyze 


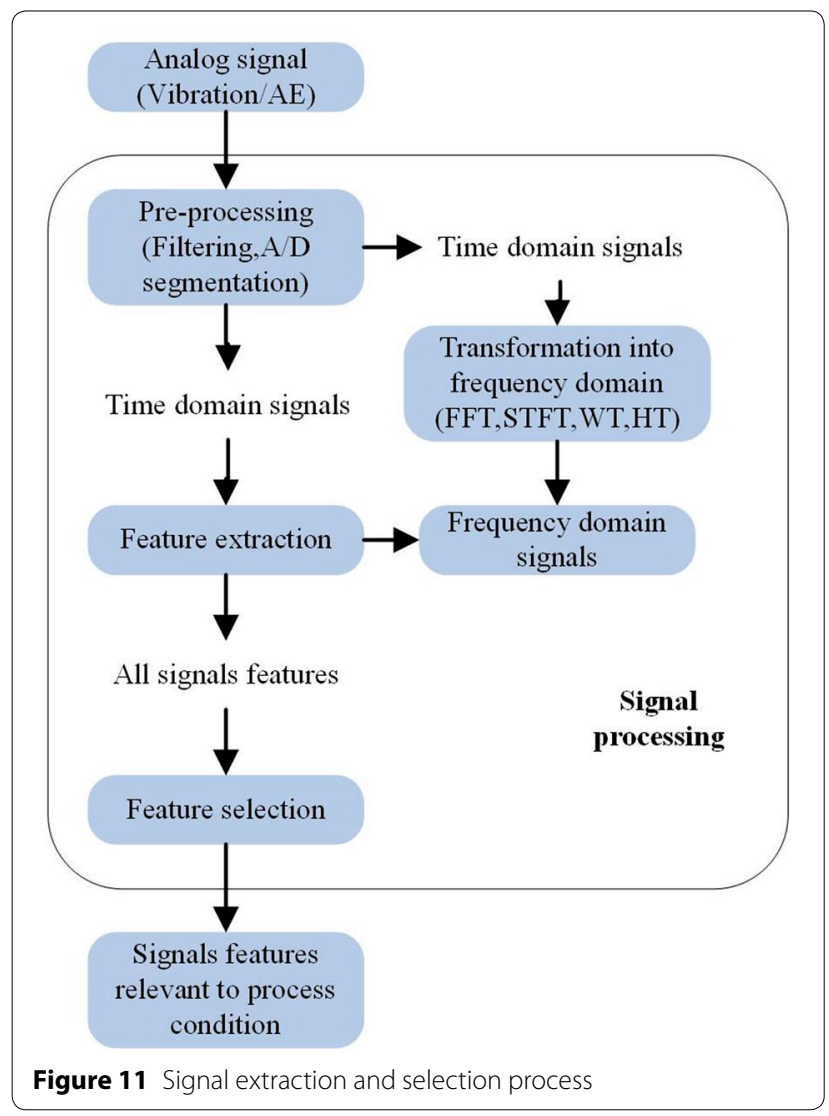

the acceleration signal of the casing and successfully identified the blade-casing rubbing fault. The aeroengine rotor test-bed is shown in Figure 12. Due to the non-stationarity of dynamics and vibration of variable speed machinery, its condition is difficult to be monitored and diagnosed. Li et al. [36] combined order cepstrum with radial basis function (RBF) artificial

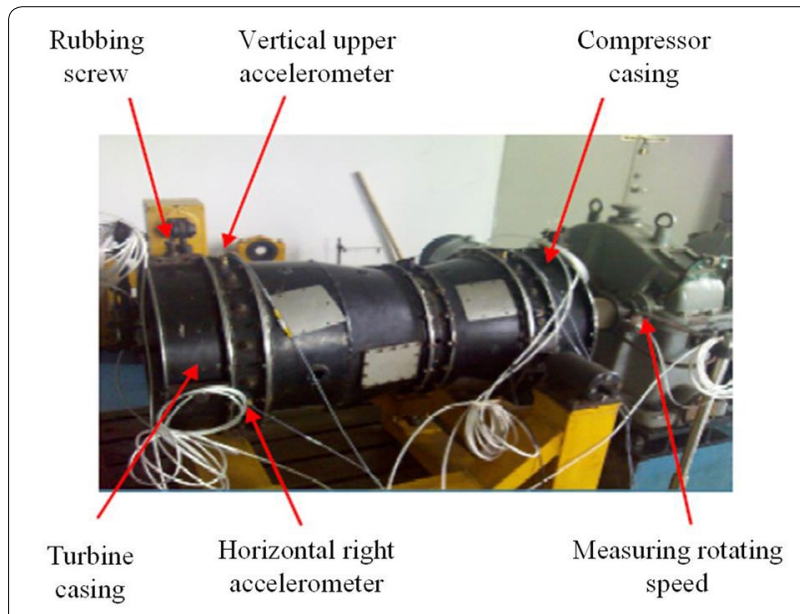

Figure 12 Aero-engine rotor test-bed neural network and applied it to gear fault detection. This method combine computational order tracking, cepstrum analysis and artificial neural network, which is effective for gear fault diagnosis.

The power spectrum (PSD) is the square of the Fourier transform (FT) amplitude of the signal, indicating the distribution of vibration power. Liang et al. [37] used the power spectrum to analyze faults of induction motors. When the faults show rich sideband and harmonic characteristics, this method has better identification ability. Jacek Urbanek et al. [38] put forward a method to extract the vibration signal components of rolling bearings which is named averaged instantaneous power spectrum (IPS). The flow chart for calculation of the averaged IPS is shown in Figure 13. Isa Yesilyurt et al. [39] proposed a method of smoothed instantaneous power spectrum, which is applied to the monitoring and analysis of gear faults.

Feature extraction based on holo-spectrum analysis is more and more widely used in rotor fault diagnosis. The traditional Fourier spectrum (FFT) has limitations in showing the whirling properties of the rotor fault (forward/reverse whirl). Patel et al. [40] used holospectrum to extract the whirling properties of the rotor to judge the fault of the rotor.

Although the frequency domain analysis can truly reflect the nature of the vibration signal, it can only analyze the stationary signal and can not reflect the change of the frequency component of the signal with time.

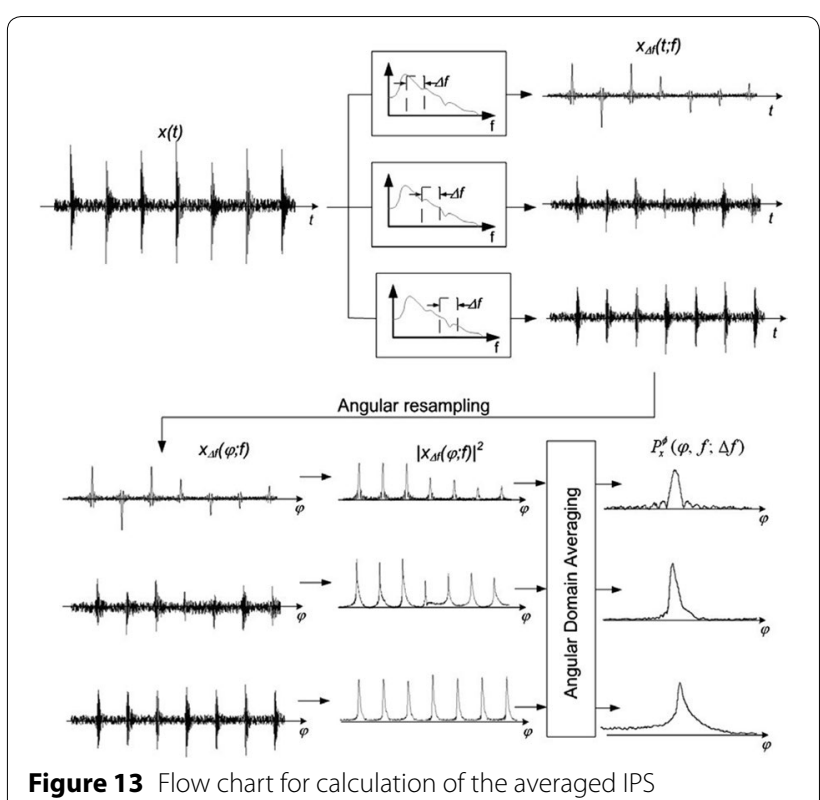




\subsection{Joint Time-Frequency Method}

In view of the advantages and disadvantages of the first two methods, researchers have proposed joint time-frequency methods, which can characterize the change of non-stationary signal frequency with time. The methods based on joint time-frequency analysis include empirical mode decomposition (EMD), short-time Fourier transform (STFT), Hilbert-Huang transform (HHT) and wavelet transform (WT).

Based on EMD method, the signal can be decomposed into limited different intrinsic mode functions (IMFs), and the feature of the raw signal in different time domain is included in every IMF component [41]. Nguyen et al. [42] used EMD to process the original vibration signal of the bearing, so as to extract feature data, and apply it to deep learning network to diagnose the related faults. Wang et al. [43] put forward a fault diagnosis method for the rotating machinery. In this method, the fault signal is processed by ensemble EMD (EEMD), and the fault is identified by self-organizing map (SOM) neural network, as shown in Figure 14. Based on the combination of EMD-ICA and Hilbert envelope demodulation, Xie et al. [44] proposed a fault information extraction method to extract fault signal of turboprop engine reducer.

Since the Fourier transform (FT) is aimed at the stationary signal, the STFT has become an important method to study the non-stationary signal. Based on STFT, He et al. [45] proposed a time-frequency feature extraction method to extract the vibration signal of helicopter rotor, and the fault detection scheme based on this method is shown in Figure 15. Jin et al. [46] analyzed the vibration signal of internal combustion engine. N. Harish Chandra et al. [47] used STFT to process the run-up data of rotor bearings, so as to diagnose the fault of shaft misalignment and rotor stator friction.

The basic process of processing non-stationary signals with HHT is to process the raw signal to get IMFs by

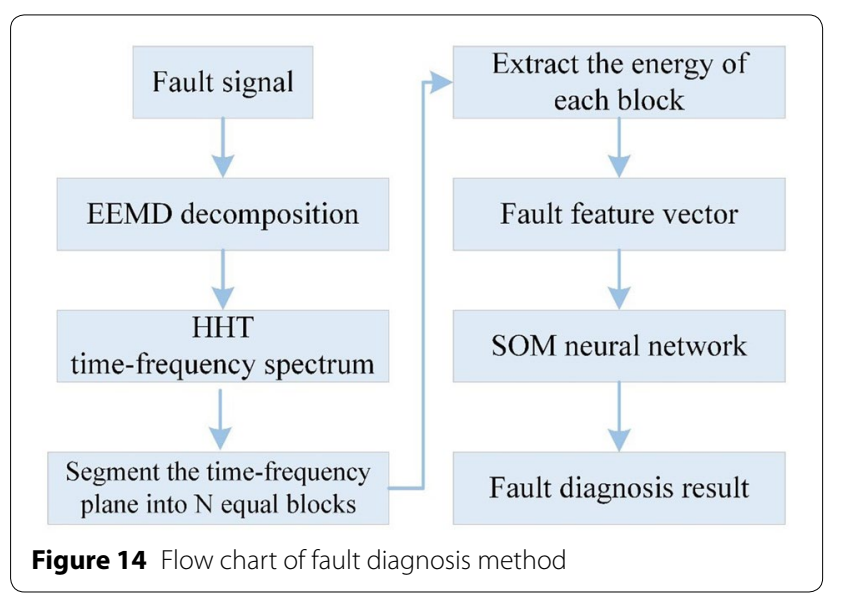

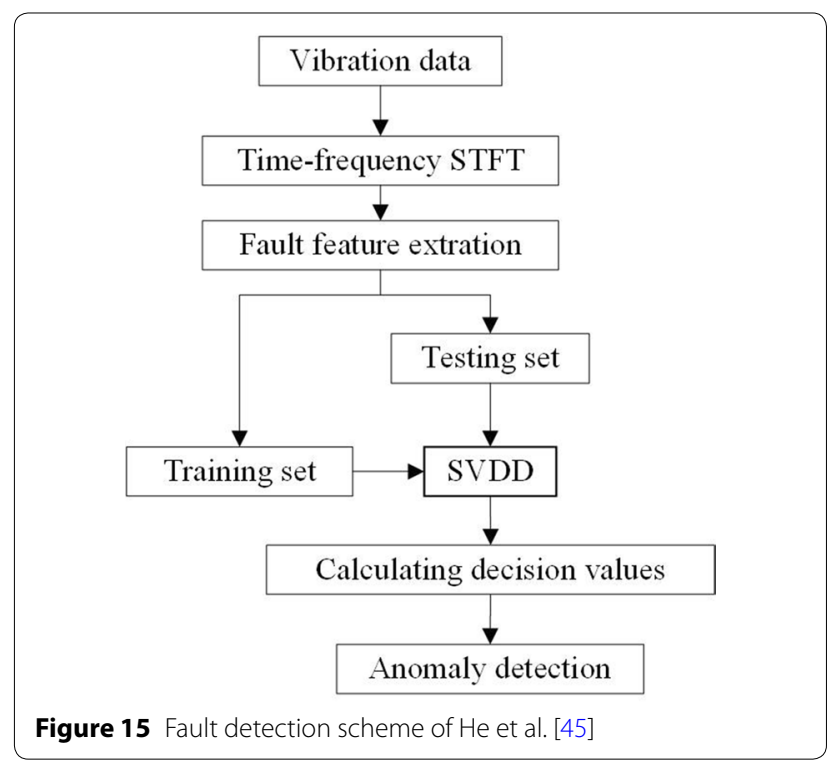

using the EMD method, and then perform Hilbert transform on every IMF to obtain the corresponding Hilbert spectrum. Li et al. [48] identified rotor cracks based on HHT.

Wavelet transform (WT) inherits and develops the concept of localization of STFT, and overcomes the disadvantage that the window length of STFT does no longer trade with frequency. The filtering characteristics of wavelet analysis in time-frequency domain are usually utilized to represent different frequency ranges of signals at different levels. Li et al. [49] proposed an adaptive harmonic window function based on wavelet transform, and the periodic signal and rotor axis trajectory can be extracted from strong noise. Fu et al. [50] utilized the improved harmonic wavelet transform to extract and analyze the vibration signals of the axis orbit, and obtained a high-resolution time-frequency picture. Muralidharan et al. [51] used continuous WT to extract fault features of centrifugal pumps, and input the feature signal into the classifier for classification. F. Al-Badour et al. [52] used the combination of continuous wavelet and wavelet packet transform to effectively extract feature signals and locate faults. Based on the integration of improved wavelet packet transform (IWPT), distance assessment technique and support vector machines (SVMs), $\mathrm{Hu}$ et al. [53] proposed a new fault diagnosis technique which can be utilized to the rotating machinery to extract significant frequency band features from the original vibration signal. Shan et al. [54] put forward a method to process the vibration signal of high-speed motorized spindle in actual running state. This method can be used to separate and extract different signal features of the motorized spindle from the strong noise by 
wavelet transform, and then analyze the frequency spectrum of the motorized spindle.

With the progress of computer technology, fault diagnosis technology is constantly developing. The feature extraction based on a single method is no longer adapt to complex and changeable working conditions. Therefore, the combination of multiple feature extraction methods is the development direction of fault diagnosis.

\subsection{Feature Extraction Based on Intelligent algorithm}

The artificial intelligence technique applied to feature extraction and fault diagnosis mainly include neural network, deep learning and other intelligent algorithms. Lobato et al. [55] proposed a diagnosis method which integrates stationary and non-stationary signal processing techniques, multi-attribute selection and classification by machine learning. According to the vibration data in the actual flight test, Hu et al. [56] of Boeing Company in the United States used artificial neural network (ANN) to detect the unbalanced state of aircraft engine. Zhu et al. [57] put forward a fault diagnosis method of convolution neural network based on symmetrical lattice images, which converts multiple vibration signals into symmetrized dot pattern (SDP) images. Then the convolutional neural network (CNN) is used to identify the SDP figure features in different vibration states. Yan et al. [58] of Harbin Institute of Technology came up with a deep belief network ((DBN)) based on multi-source heterogeneous information fusion for special diagnosis and diagnosis of rotor unbalanced faults. The experimental results show that under the appropriate DBN parameters, the accuracy of feature extraction and fault classification of rotor unbalanced faults reaches $100 \%$. Figure 16 shows the fault diagnosis process of this method. Shao et al. [59] proposed a deep learning method based on deep belief network (DBN), which is utilized to learn features from the frequency distribution of vibration signals and to identify fault states. Figure 17 shows the DBN-based troubleshooting steps. Chen et al. [60] established a vibration fault diagnosis model based on the combination of cuckoo search algorithm with memory (CSM) and BP neural network. The faults such as rotor unbalance and

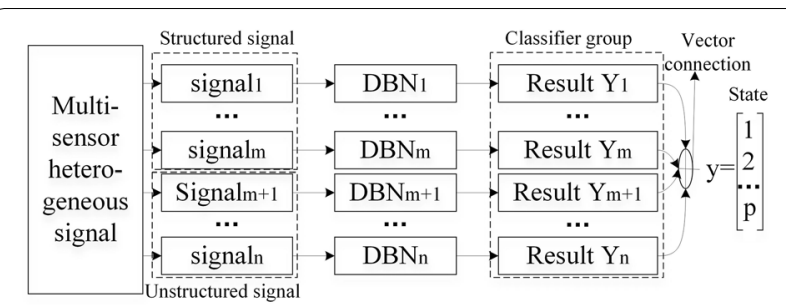

Figure 16 Fault diagnosis process of DBN based on multi- source heterogeneous information fusion

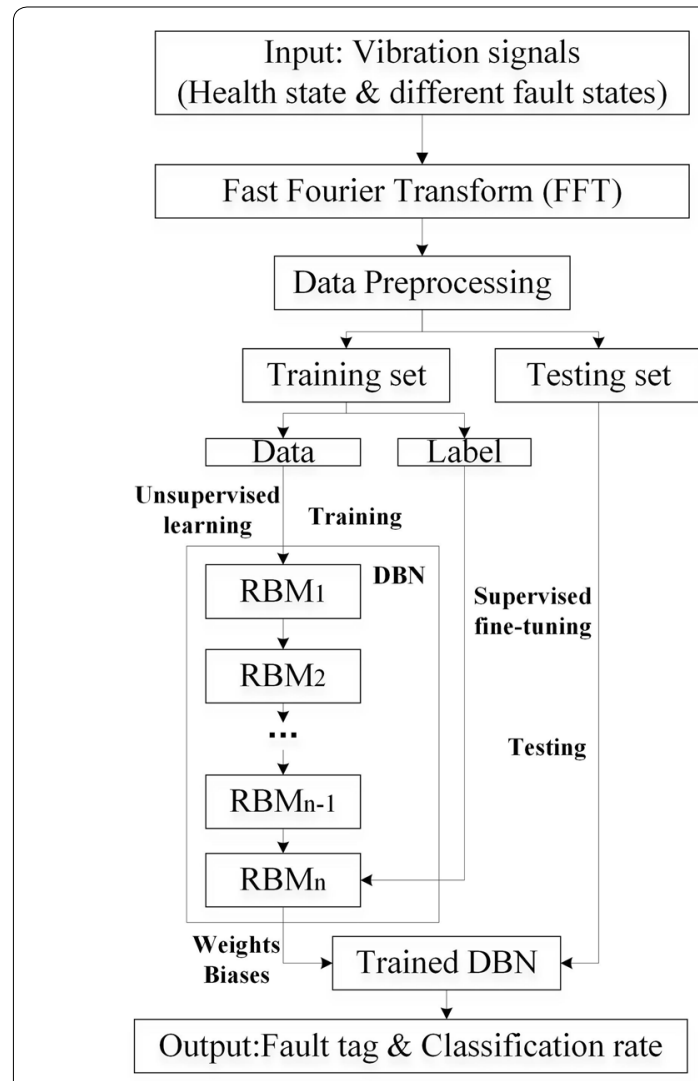

Figure 17 Deep learning method based on DBN

rotor misalignment diagnosed by this method are consistent with the actual fault types. Li et al [61] of the University of Alberta in Canada came up with an adaptive tracking technique based on sinusoidal synthesis, which uses the sinusoidal characteristics of vibration signals to transform a nonlinear problem into a time-domain linear adaptive problem based on state space. $\mathrm{Lu}$ et al. [62] put forward a genetic algorithm based on dynamic search strategy, which showed good feature extraction and selection ability in two kinds of fault experiments of rotor unbalanced vibration and bearing damage. Deng et al. [63] presented an improved classification and regression tree (CART) algorithm, namely D-CART algorithm, which can quickly locate the rotor system fault and identify the fault type on the premise of ensuring the accuracy.

In summary, with the development of computer technology, feature extraction and fault diagnosis technology has developed rapidly from traditional signal processing technology to intelligent direction, which lays a foundation for the application of SR Technique. However, the feature extraction technique based on neural network, deep learning and other intelligent algorithms is still in the stage of development, and a series of bottlenecks 
need to be solved, such as the training difficulty and fitting ability of the model, the real-time performance of signal analysis and extraction, and so on. These are also the urgent problems that need to be solved in the future.

\section{Imbalance Location}

The imbalance location belongs to the second step of fault diagnosis in the SR system for unbalance vibration, and it is the key to the targeted control of unbalance vibration and the execution of self-recovery. At present, there are mainly three kinds of methods to locate the imbalance of the rotor system: (1) Influence coefficient method, (2) Modal method, (3) Holographic dynamic balancing method [64-68].

\subsection{Influence Coefficient Method}

The core of this method is to acquire the influence coefficient matrix, which is used to express the linear relationship between the vibration response and imbalance on several specific planes of the rotor. While the influence coefficient matrix is known, the initial imbalance of the rotor can be located by dividing the vibration response matrix and the influence coefficient matrix $[69,70]$.

Goodman et al. [71] made a comprehensive discussion on the influence coefficient method in 1964 and put forward its general expression for the first time. In addition, they introduced the least square method into the influence coefficient method to calculate the final correction mass of multi-speed and multi-plane balance of rotating machinery. Kang et al. [70] obtained better balance accuracy by reducing the number of equilibrium conditions. Lei et al. [72] put forward an improved influence coefficient method for anisotropic rotor bearing systems, which uses the amplitude and phase of the forward precession instead of the traditional unbalanced response, and can obtain the similar result with the conventional method. Considering the randomly distributed residual unbalance and the residual unbalance of the balance disk, Ranjan et al. [73] proposed a general influence coefficient method which is suitable for the active magnetic bearing rotor system. It can ensure the safe operation of unbalance rotors at high speed by use of active magnetic bearings. Fujisawa et al. [74] studied a multi-plane multi-speed balance method, which uses the least square method of influence coefficient and takes into account the vibration characteristics of multi-span rotors to calculate the correction amount.

For the complex and changeable high-speed rotor system, the fast and accurate calculation of imbalance is regarded as an important factor affecting the balance effect. Kang et al. [75] researched the dynamic balance of uneven rotor-bearing system. On the basis of the motion equation of asymmetric rotor, the influence coefficient matrix expression of asymmetric rotor was derived by way of the use of complex coordinate illustration and finite element method. Moon et al. [76] proposed to use the reference influence coefficient method of the reference model for active balancing, and tested the method on a machine tool. The balance experimental device is shown in Figure 18. By using this method, the vibration is reduced to $54 \%$ near the critical speed, the reduction effect in other parts is more than $80 \%$, and the average reduction effect is $70 \%$ of the initial unbalance response. According to the actual conditions of dynamic balancing in the field, Untaroiu et al. [77] put forward a general optimization, that is, to minimize the maximum residual vibration of dynamic balancing with influence coefficient. The technique can be easily used in all rotor balancing methods that using correction amounts, and even to active balancing. When the two-plane influence coefficient method is used in dynamic balancing, it does not need an excessive amount of statistics of the rotor system, and it can gather and process data automatically. Xu et al. [78] used the two-plane influence coefficient method to balance the rigid rotor, and the vibration reduction rate of the rigid rotor can reach more than $85 \%$ after one dynamic balance correction.

For the turbomachinery with complex deformation, multiple constraints and less measurement information, Bin et al. [69] put forward a high-speed balancing method for $\mathrm{N}+1$ supported turbomachinery without trial weight, which can improve the balancing efficiency and accuracy of the rotor. Based on the multi-plane influence coefficient, this method can determine the relationship between the synchronous vibration and the unbalance excitation. It is based on the prediction of the steadystate response of the finite element method to calculate the multi-plane influence coefficients at several rotational

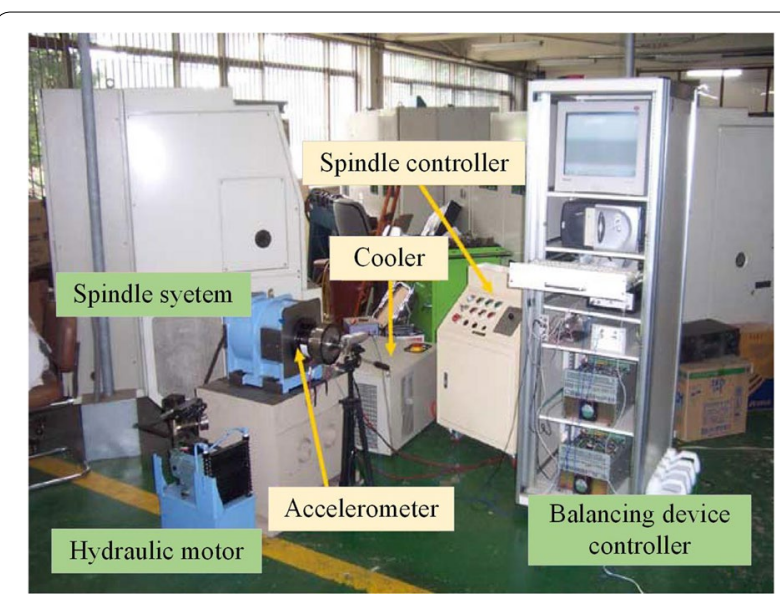

Figure 18 Balance experimental device of Moon et al. [76] 
speeds. The experimental device and imbalance location results are shown in Figure 19. Through the experimental verification, the bearing vibration is reduced by an average of $25 \%$ and the maximum is reduced by $50 \%$ at 2700 $\mathrm{r} / \mathrm{min}$.

\subsection{Modal Method}

The method is to utilize the modal characteristics of the unbalance vibration of the rotor, decompose the unbalance according to each order of modals, correct the imbalance respectively, and finally restrain the unbalance vibration of the rotor. Compared with the influence coefficient method, this method is more sensitive to higher vibration modals.

Han et al. [79] studied a new type of generalized modal balance of isotropic rotor systems. In this method, the unbalance modal response is obtained by analyzing the modes of non-isotropic rotor systems. It was simulated to verify its theoretical results and effectiveness. Because the low pressure rotor of large steam turbine is difficult to be balanced by the traditional influence coefficient method, based on modal analysis, Bin et al. [80]

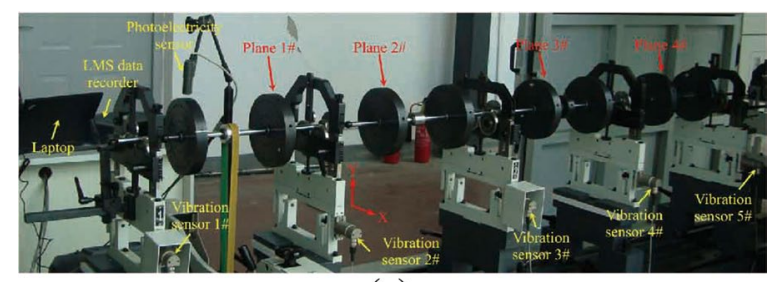

(a)

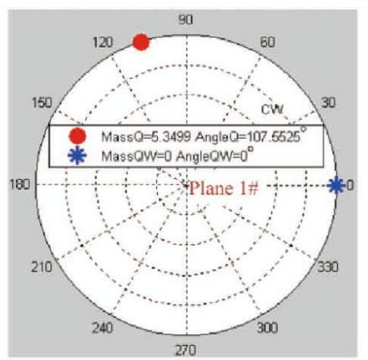

a plane $1 \#$

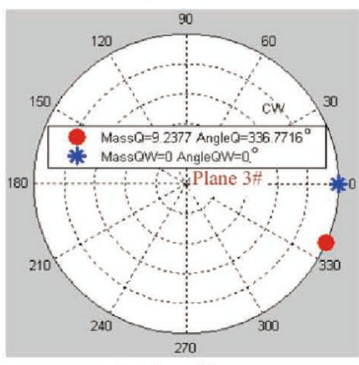

c plane $3 \#$

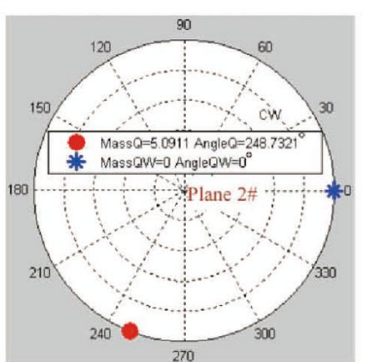

b plane $2 \#$

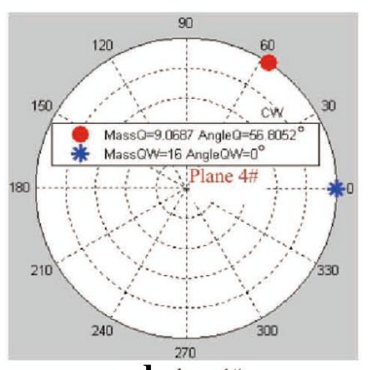

d plane $4 \#$ (b)

Figure 19 Imbalance location results of Bin et al. [69]: a Experimental device; $\mathbf{b}$ Imbalance location results put forward a high speed balancing method of large flexible rotor, so as to realize the dynamic balance of this kind of large special flexible rotor. Quartarone et al. [81] improved the modal balance method and put forward a method to identify the balance mass group of high-speed rotors, which allows one critical speed to be balanced selectively at a time and minimizes the impact on the unbalanced response in the rest of the speed range. This method is used to balance the turbine molecular pump and its vibration is significantly reduced.

\subsection{Holographic Dynamic Balancing Method}

In view of the disadvantage that the traditional spectrum analysis only pays attention to the amplitude-frequency relationship while neglecting the phase information, the holographic spectrum theory is put forward to overcome the shortcomings of the former two methods by means of dual-sensor measurement [82].

Liu et al. [83] proposed Low-Speed Holo-Balancing method to balance a flexible rotor without test runs at high speed. The variation of the first two modal components of the rotor during the start and stop phase is analyzed by using the decomposition of the holographic spectrum, and the first two low-mode components can be offset together when the speed is lower than the first critical speed. Based on FFT spectrum and information fusion, the holographic spectrum technique was used to the dynamic balancing of flexible rotor systems, and holographic balancing method was proposed [84]. Combined with computer simulation and genetic algorithm, this method simplifies the dynamic balancing process and improves the speed of unbalance and phase recognition, and its effectiveness was validated by balancing of 300 MW turbo-generator units shown in Figure 20.

In summary, the imbalance location method is mainly based on the above three methods, and other methods are improved and optimized on the basis of them. Whether the position of the unbalance and the size of the unbalance can be accurately discriminated is the key to the implementation of the follow-up adjustment method to restrain the vibration. Therefore, the rapid positioning of unbalanced vibration and high-precision calculation of unbalance are important research content in the future.

\section{Regulation Method}

During the dynamic balancing process, the vibration signal is processed by the controller, and the resulting vibration amplitude is used to determine whether it exceeds the threshold set in advance. If it exceeds the limit, the controller will continuously output control instructions to the actuator according to the corresponding regulation methods. Therefore, the regulation method has a 

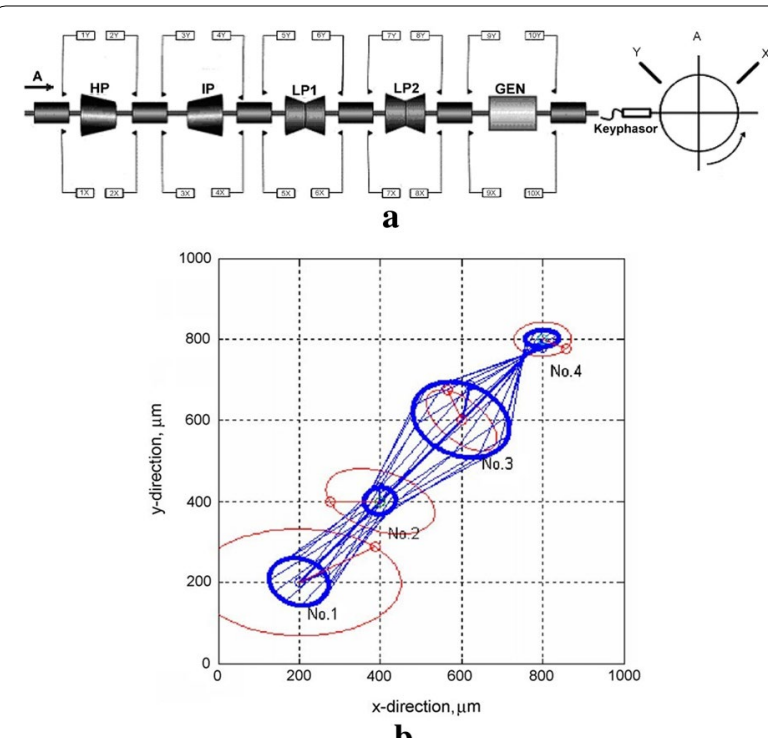

b

Figure 20 Balancing results of Liu et al. [83]: a Structure of turbo-generator; $\mathbf{b}$ Balancing results

significant impact on the balance accuracy and efficiency of the balance system.

In actual situations, the system structure often has complicated nonlinear dynamic characteristics. Therefore, the regulation method needs to be based on the system dynamics model, using modern or intelligent control theory to design. At present, pole assignment method, optimal control and intelligent control are the commonly used control strategies.

\subsection{Mechanism of the Exoskeleton Arm}

The dynamic performance of pole assignment system is principally determined by the distribution of poles on the foundation plane. Once the state area expression or transfer operate of the system is understood, the pole of the control system is affected to the selected position by adding the state feedback controller [85]. For asymmetric systems, Ariyatanapol et al. [86] projected the pole-delay methodology of the time-delay half exploitation singleinput state feedback management, which may accurately piece the specified closed-loop points while not dynamic different poles. Based on $\mathrm{H} \infty$ controller and feedforward compensation controller. Real-time online force automatic compensation was implemented by Gao et al. [87]. Combined with the pole placement theory, Liu et al. [88] proposed an improved hybrid $\mathrm{H} 2 / \mathrm{H} \infty$ control technique and achieved the synchronous management of attitude stabilization and vibration suppression for versatile satellite. Fan et al. [89] projected an adaptive observer-based state-feedback management technique for stabilization of the integrated fault designation and mechanism fault-tolerant management systems subject to regulate input constraints. As shown in Figure 21, the initial feasible solution of nonlinear optimization is obtained by pole placement method to improve system performance.

\subsection{Optimal Control Method}

Optimal management theory was shaped and developed below the impetus of space technology. For a controllable dynamic system or motion method, the optimum control theme is pointed out from all control schemes, so once the motion of the system is transferred from an initial state to a specific target state, its performance index price is perfect. The rotor imbalance will cause synchronous vibration of magnetic levitation centrifugal compressor system. As shown in Figure 22, a synchronous vibration management methodology associate in nursing optimum notch filter was planned by Peng et al. [90], which stabilized the synchronous vibration force in the full speed range. Based on the classical optimal control theory, Tombul et al. [91] simplified the nonlinear control problem to linear control, and obtained the nonlinear optimal control law through the sequence of linear time-varying systems. When the shaft is rotating at a constant angular velocity, this method enables fast and robust control of unbalanced vibrations.

Rosyid et al. [92] applied the optimal control method to the control of the rotor-bearing-support system with a large degree of freedom. Two pairs of different weighting

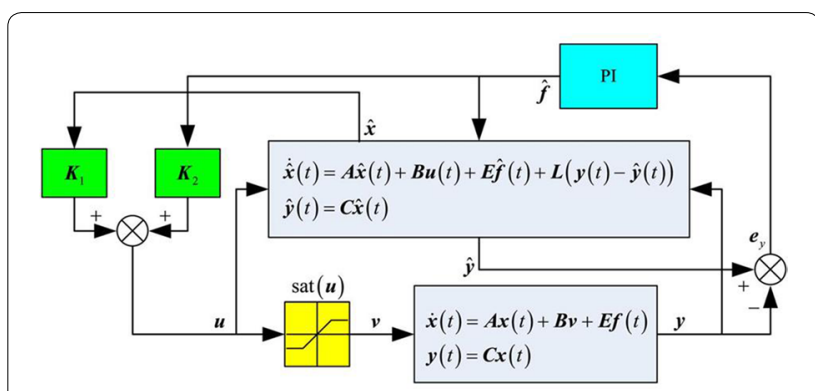

Figure 21 Block diagram of closed-loop system

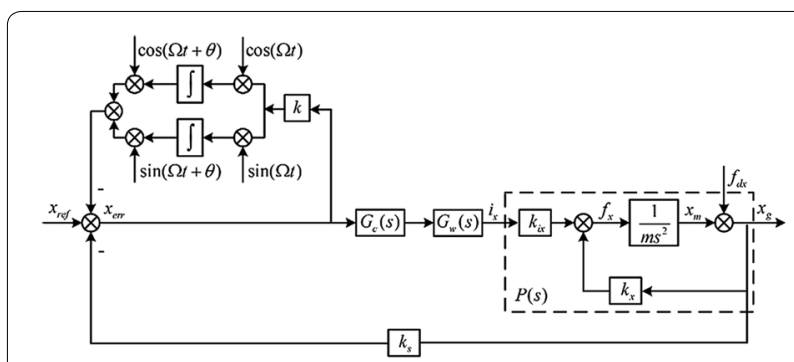

Figure 22 Bock diagram of MSR system synchronous vibration management supported section shift notch filter 
matrices were used to control the unbalance vibration of low-speed and high-speed rotors respectively through a linear quadratic regulator. A model prophetic best management methodology for magnetically suspended regulator is bestowed by Zhang et al. [93]. The optimum controller is obtained by means that of iterating a Riccati distinction equation, and stability of the management theme is investigated through faux algebraical Riccati technique. Simulations and experiments show that the controller will effectively suppress the cone-shaped vortex K.E. caused by the gyro effect. As shown in Figure 23, Yang et al. [94] established an optimal control model for controlling the vibration of a rotor system of a tilting pad gas bearing using piezoelectric actuators, proportional differential compensation and gap sensors. The optimal control theory was used to obtain the optimal control rules between the actuator voltage and the dynamic displacement of the rotor, and the results proved that the optimal control system could effectively suppress the unbalanced response of the system.

\subsection{Intelligent Control Method}

For the optional control method, the determination and selection of the optimal control objective function is complicated. With the event of intelligent technology, intelligent algorithmic program are wide employed in the regulation system. Carvalho et al. [95] introduced the fuzzy algorithm to improve the traditional influence coefficient method, and the robustness of vibration control was improved through fuzzy processing of rotor vibration response. Xu et al. [96] proposed a fuzzy self-tuning single neuron PID control method, and the schematic

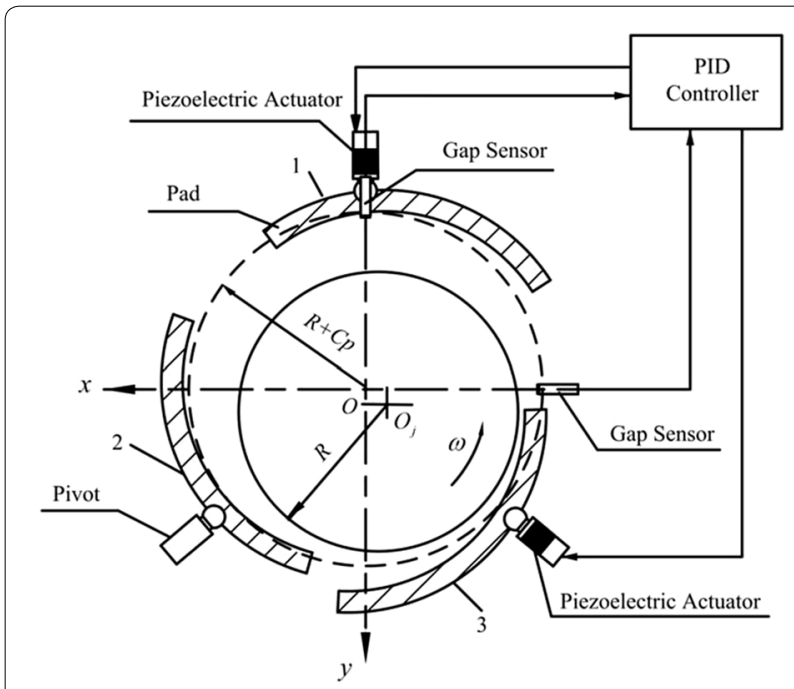

Figure 23 Active control model of rotor-bearing system established by Yang et al. [94] diagram is shown in Figure 24. Compared with ancient inflammatory disease and single somatic cell inflammatory disease management ways, it has several characteristics, such as fast response time, small overshoot, few oscillations, strong robustness, and good stability. Oke [97] applied evolution algorithm to reduce the residual imbalance of the rotor system. The results show that the algorithm is superior in optimizing time and effectively reducing vibration values. Yang [98] considered the discrete distribution of the unbalance mass and phase, and projected a hybrid genetic algorithmic program with neighborhood search. The influence of the difference between theoretical calculation and the actual addition of the balance weight was avoided, and the balance accuracy and speed was improved. Yao et al. [99] improved the PID algorithm with fuzzy control, as shown in Figure 25, and the minimum mean square filter was connected to the fuzzy control to compensate the unbalanced quality. Simulations show that the improved fuzzy PID formula performs higher than ancient PID management in overshoot management, and also the transient time of fuzzy PID is considerably shortened.

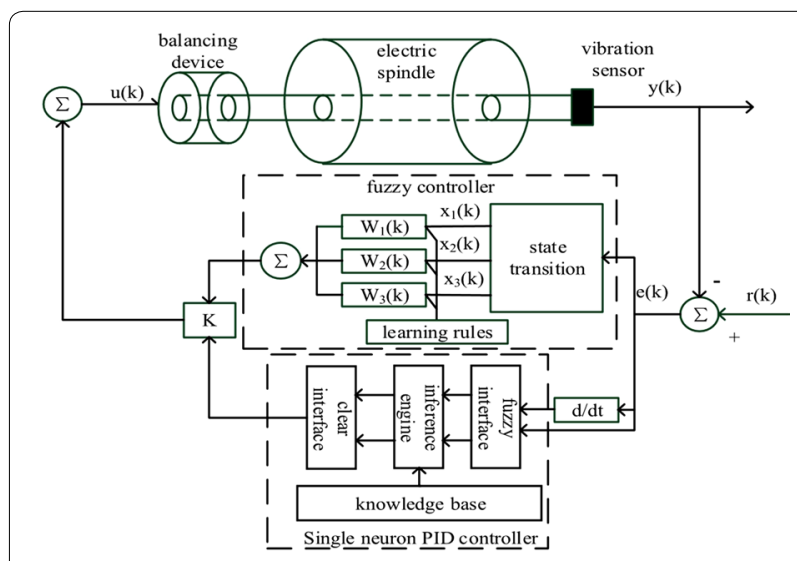

Figure 24 Schematic diagram of fuzzy self-tuning single neuron PID control

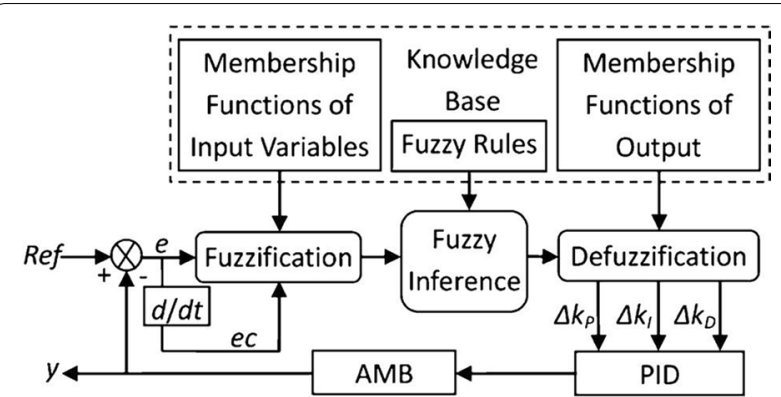

Figure 25 Block diagram of fuzzy PID control scheme by Yao et al. [99] 
The selection and design of automatic balancing control strategy for complex rotor system need to be determined according to actual working conditions and specific actuators. Simple algorithm, good working stability, high balance accuracy and high balance efficiency are important characteristics for evaluating regulation methods. In the future, the development trend of automatic balance control methods is to combine the reliability of traditional control methods with the high efficiency of modern intelligent control methods to deal with the self-recovery regulation control of unbalanced vibration under different working conditions.

\section{Balancing Actuator}

For the balancing process of online dynamic balancing, the actuator accepts the control instructions issued by the controller and acts according to the corresponding regulation method to achieve online dynamic balancing.

As the executive part of the self-recovery regulation system, the structural design of actuators is the focus of self- recovery research. For the unbalance vibration, the actuator is called as balancing actuator, balancing head, or simply as balancer, and the performance of the balancing actuator directly determines the final balance effect of the self-recovery system. According to the balancing principle, the balancing actuators can be divided into active type and passive type, as shown in Figure 26. For the passive type, an obtuse angle is formed between the rotor deflection response and the delayed unbalanced excitation force in the supercritical state of the flexible rotor, which allows the balancing actuator to automatically adjust the mass distribution for balance purposes. Based on the difference of structure and balancing medium, the passive type can be subdivided into hydraulic type, ball type and pendulum dampers type [100]. For the active type, the internal mass distribution changes according to the control commands of the controller. The

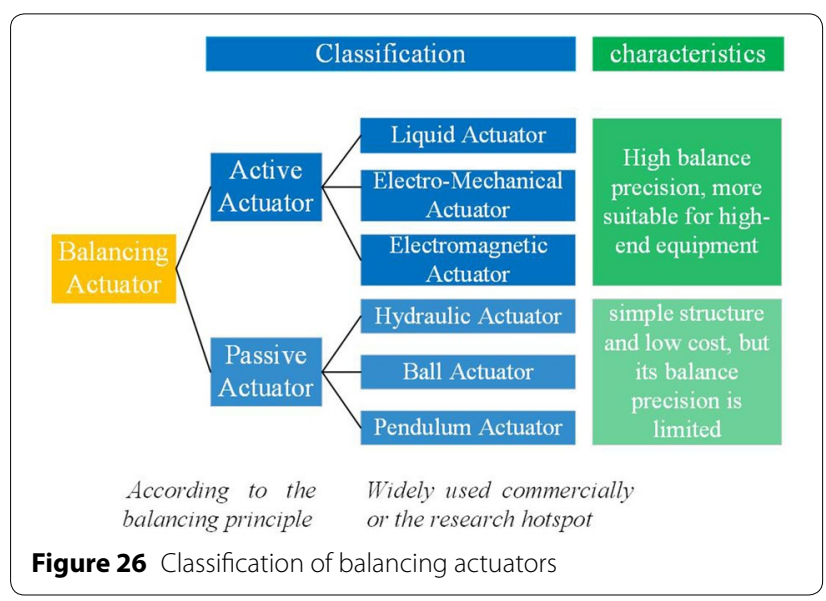

specific form is as follows: liquid type, electro-mechanical type and electromagnetic type [101-103].

\subsection{Passive Actuator \\ 7.1.1 Hydraulic Actuator}

As shown in Figure 27, the hydraulic balancing actuator is used for automatic balancing of washing machines. The balancing device is composed of the annular cavity filled with fluid and the baffle. The fluid rotates in reverse and interacts with the baffle in the cavity to generate radial and lateral circulating waves. When the hollow rotor is fitted with appropriate baffles, the unbalance of the rotor can be offset by the force generated by the fluid in the hollow rotor [104]. However, the application conditions of this self-balancing device are affected by rotor damping, stiffness, fluid viscosity and fullness, etc. $[105,106]$.

\subsubsection{Ball Actuator}

When a mass imbalance occurs at a high-speed rotating rotor, the rotor's main axis of rotation and its inertia axis are inconsistent, resulting in excessive radial vibration and severely affecting the safety of the operation of rotating equipment. Ball actuator is composed of a circular channel around the rotor and a rolling ball inside it. Under the action of the centrifugal force generated by the high-speed rotating rotor, the rolling ball falls at an angle of $180^{\circ}$ with the rotor imbalance. The imbalance is canceled, and the radial vibration is significantly reduced. This kind of dynamic balancing actuator has been well applied in hard disk and optical disc drives, and can usually be divided into single ball type [107] and double ball type [108]. A double-ball automatic balancing actuator is shown in Figure 28.

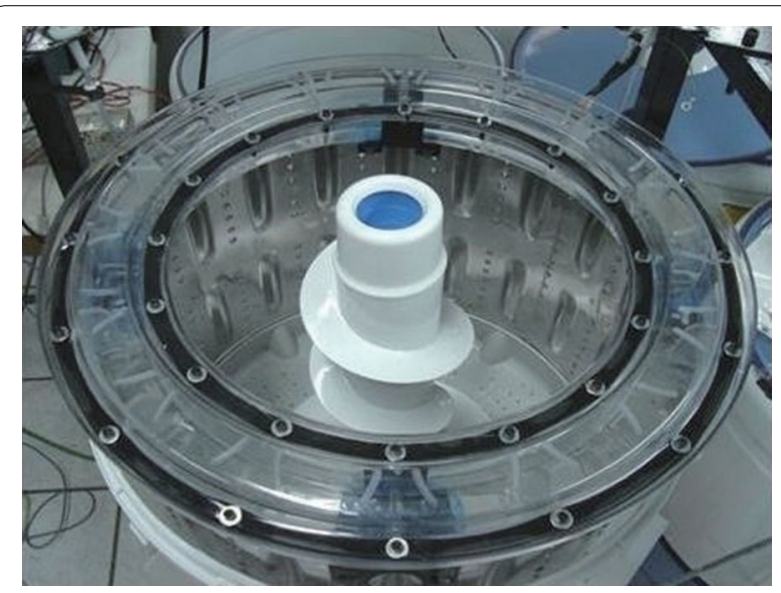

Figure 27 Balancing actuator for a washing machine 


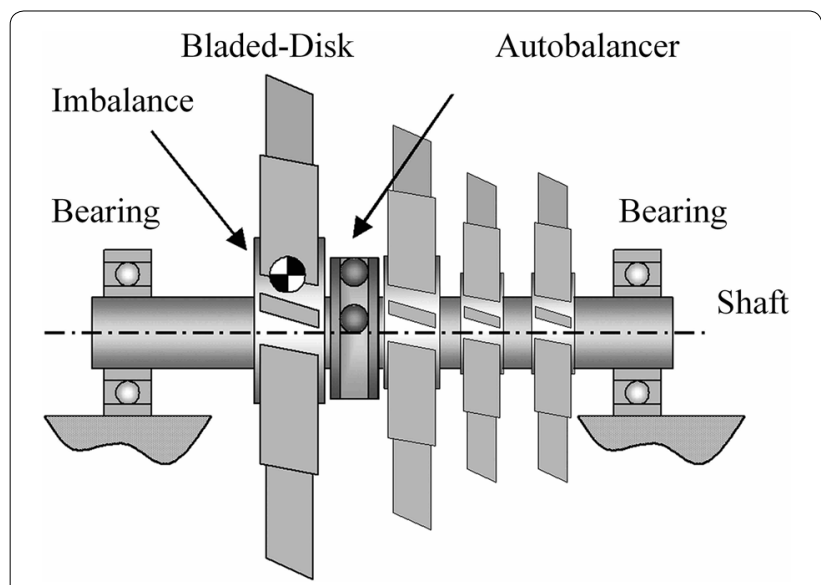

Figure 28 A double-ball automatic balancing actuator

\subsubsection{Pendulum Actuator}

The pendulum-type automatic balancing device is suitable for flexible unbalanced rotors with elastic support. The pendulum is mounted on the disc in a hinged manner. The flexible rotor system drives the pendulum to move in response to the formed energy, causing the pendulum to rotate, which in turn affects the vibration of the unbalanced rotor $[109,110]$. However, the device requires the rotor to be elastically supported, and the scope of application is small. The dynamic model of the pendulum balancing actuator is shown in Figure 29.

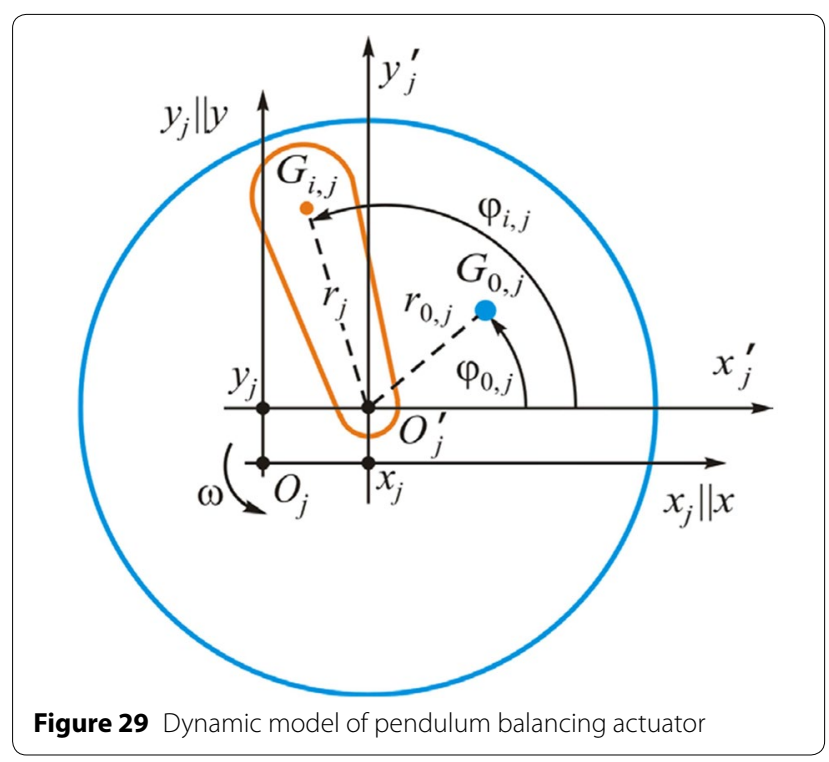

\subsection{Active Actuator}

\subsubsection{Liquid Actuator}

There are multiple liquid storage chambers inside the liquid balancing actuator. A certain amount of liquid is injected or discharged in one or several liquid storage chambers to realize the mass redistribution of the balancing actuator to compensate the original imbalance.

The liquid-injection type balancing device produced in German has been used in high-speed and ultra-high grinding machines, and is mostly composed of three or four independent fan-shaped liquid storage chambers, as shown in Figure 30. Each liquid chamber is provided with a water inlet channel on different radius. Each water inlet channel corresponds to a liquid injection nozzle, liquid injection pipe and liquid injection solenoid valve. The time of liquid injection is controlled by solenoid valve, then the mass and distribution of the liquid in the balance plate are changed, and the unbalance of the rotor is compensated.

$\mathrm{Su}$ et al. [111, 112] built a rotor test-bed to verify the two-sided dynamic balance experiment of the actuator, and applied the actuator to the actual fan rotor. Zhang et al. [113] improved the shape of the liquid chamber and developed a high-speed spindle on-line dynamic balancing device, which was assembled in the form of an interference fit between the inner and outer rings. The experimental equipment is shown in Figure 31. The liquid balancing device performed balancing operation when the spindle speed was $20700 \mathrm{r} / \mathrm{min}$, and the unbalance amplitude decreased by $78.8 \%$. Using 3D printing technology, an integrated balancing actuator was made by Mei et al. to avoid the assembly of the inner and outer rings, and the reliability was verified by the online balance test [114].

A balancing device for centrifugal spinning machine was proposed by Martin et al. and has a similar working principle to the liquid injection type. The balancing device rotates with the rotating shaft and has multiple

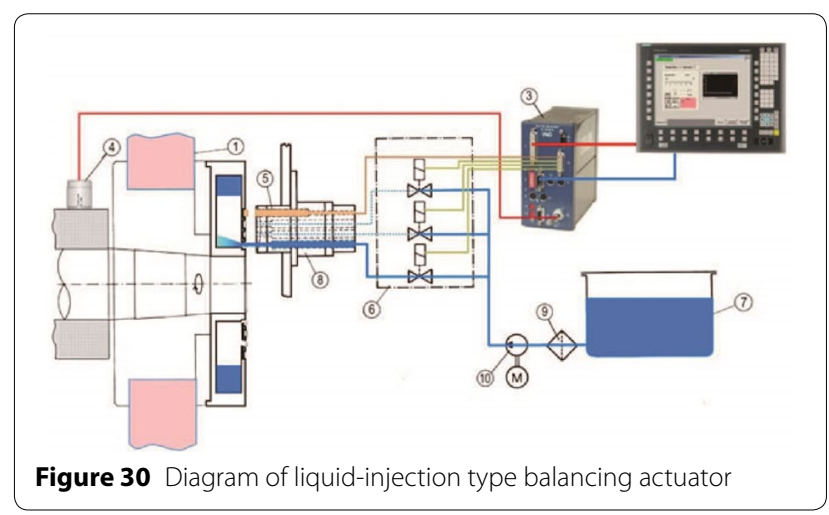




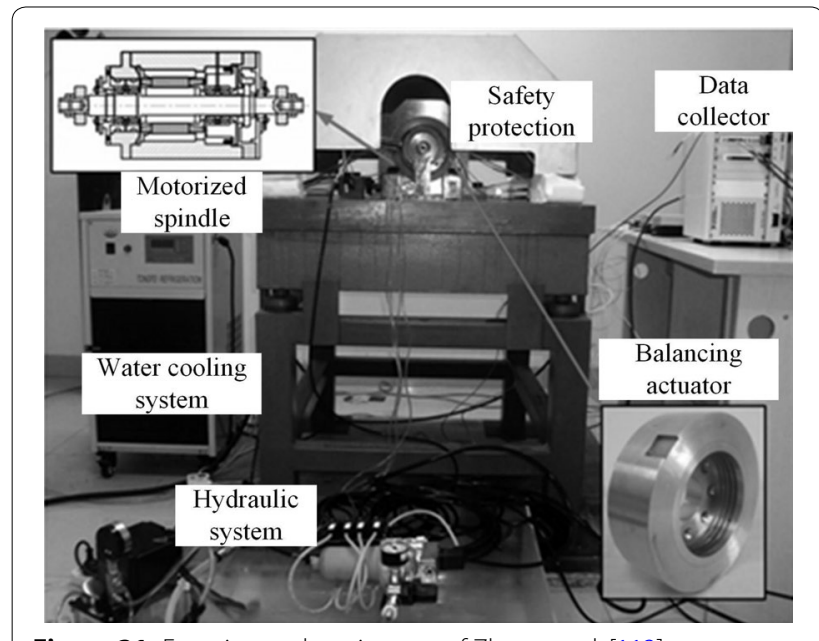

Figure 31 Experimental equipment of Zhang et al. [113]

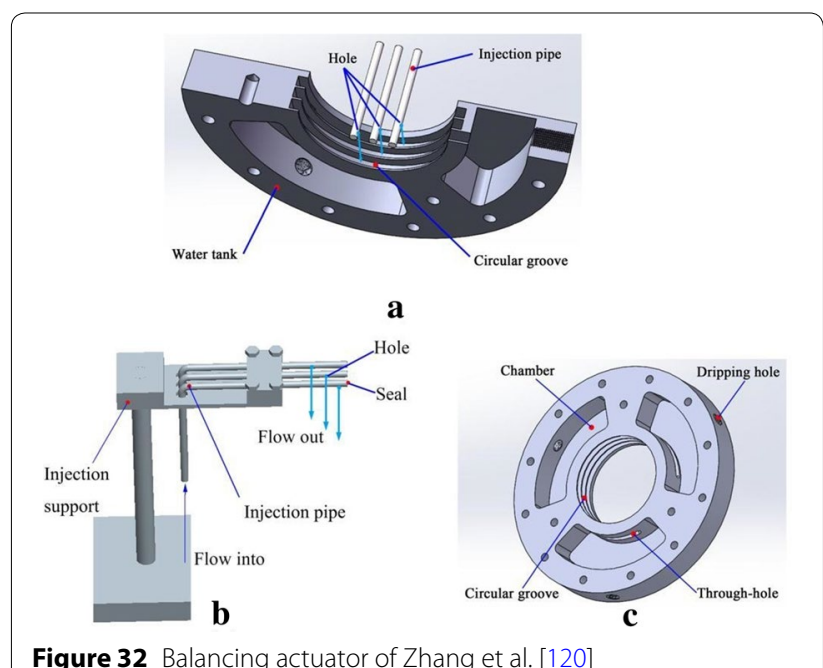

Figure 32 Balancing actuator of Zhang et al. [120] liquid chambers around the rotating shaft and a liquid distribution unit. When balancing is needed, the liquid distribution unit can inject the balancing liquid into one or more liquid chambers [115].

For the above liquid-injection type, the liquid cannot be released during the machine running, and the liquid chamber will be filled with liquid after several balancing operation. Therefore, He [116] proposed a liquid release balancing device, in which the balancing liquid can go into the liquid chamber equally and the unbalance can be component through the liquid release from the chamber. Gao et al. [117] developed a continuously dripping liquid-injection balancing device, the balance ability of which cannot be reduced by dripping liquid. The device was installed on a high gravity machine by $\mathrm{Li}$ et al. and it was verified that the actuator can maintain the vibration below $50 \mu \mathrm{m}$ within $40 \mathrm{~s}$ [118]. A pumped liquid type balance device for a fan was designed by Rumin et al. [119] and can also realize the function of injecting and draining liquid. A certain quantity of liquid may be injected into the acceptable chamber employing a pump. If the amount of balancing liquid needs to be reduced, an appropriate amount of liquid is released from the chamber using centrifugal force. Zhang et al. [120] designed a controllable injection and free dripping balancing devices for grinding wheel. The liquid injected into the balance chamber was controlled, the liquid dripped from the balance chamber can be calculated to control the residual liquid mass in the balance chamber, and then the residual liquid was used to provide a proper balance correction amount. According to the test results, dynamic balance was achieved at a grinding wheel speed of $2700 \mathrm{r} / \mathrm{min}$, and the amplitude decreased by $89.25 \%$. The structure of the actuator was shown in Figure 32.
To solve the liquid splashing during liquid injection and failure to maintain balance after shutdown, liquid transfer actuators are invented to avoid the liquid injection process. Steere et al. [121] designed the heating actuator and a vaporizable liquid was pre-filled in a plurality of circumferentially spaced chambers. During the balancing process, the heating elements in selected chamber were activated and the vaporized medium flowed into the adjacent chamber to balance the initial imbalance. Coyne et al. [122] used pumps rotating with the balance device to realize the balance liquid transfer between the relative liquid storage chambers. A oneway pump and a spool line or two one-way pumps and two solenoids were required for each pair of chambers. Nakamoto et al. [123] projected a balancing actuator using magnetic fluid, in which magnetic field dominant module and reconciliation disc were organized individually. Based on the similar mechanism, Zhang et al. [124] introduced an integrated style between the dominant module and reconciliation disc.

Although the above-mentioned liquid transfer balancing devices get rid of the process of liquid injection, there are movable parts in the rotating actuator, so they are not suitable for high-speed occasions. To solve the problem, Pan et al. [125] developed a pneumatic liquid balancing actuator with no movable parts in the rotating part, and compressed air was used to drive the balancing liquid transfer between opposite liquid chambers. The experimental setup is shown in Figure 33, and the unbalance vibration of the rotor is effectively suppressed within $10 \mathrm{~s}$ at the speed of 15600 $\mathrm{r} / \mathrm{min}$, and the amplitude is reduced by more than $80 \%$ [126]. 


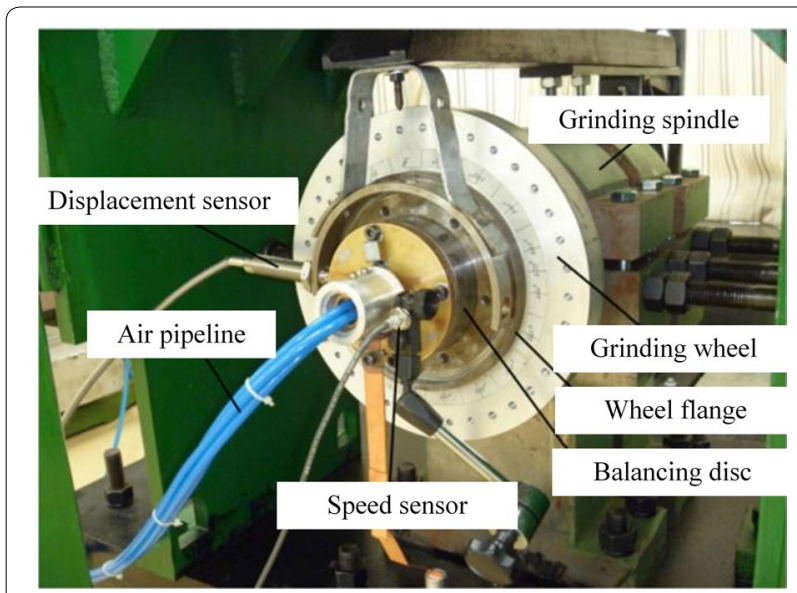

Figure 33 Experimental setup of pneumatic liquid actuator

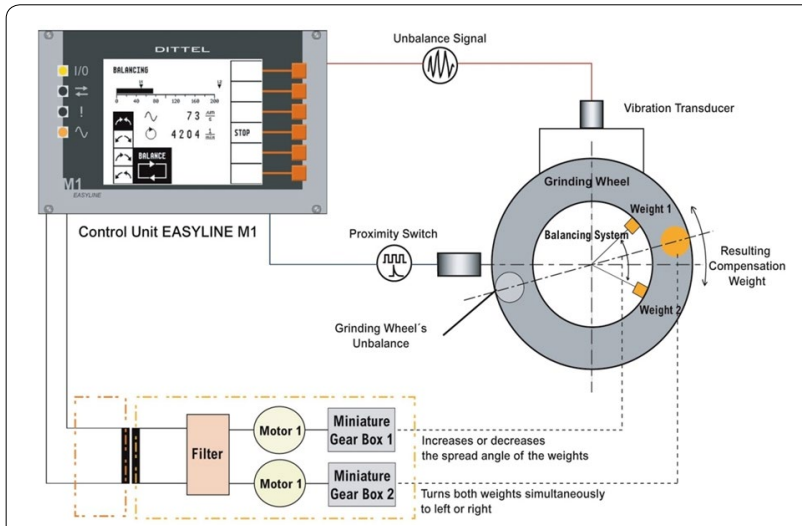

Figure 34 Balancing principle of electro-mechanical actuator

\subsubsection{Electro-Mechanical Actuator}

As shown in Figure 34, this type actuator achieves mass distribution through a pair of position-controllable counterweights, each counterweight is driven by a motor and a gear box, and the angle between them is adjusted to generate the compensation weight [127].

Vegte et al. [128] used two motors powered by the slip ring of carbon brush to drive two counterweights respectively. Kim and Lee [129] realized the balance head control using radio remote control, and achieved on-line automatic dynamic balancing of the flexible rotor above the first-order critical speed. Li and Lu [130] designed the permanent magnet difference frequency (PMDF) motor to realize the contactless transfer of control signal and driving energy between the control unit and the balancing actuator. Hredzak et al. [131] cancelled the motor and placed steel balls into ring-shape grooves to vary the balancing status.

As mentioned in Section 3.2, this type balancing actuator has been widely used in grinding machine, and several

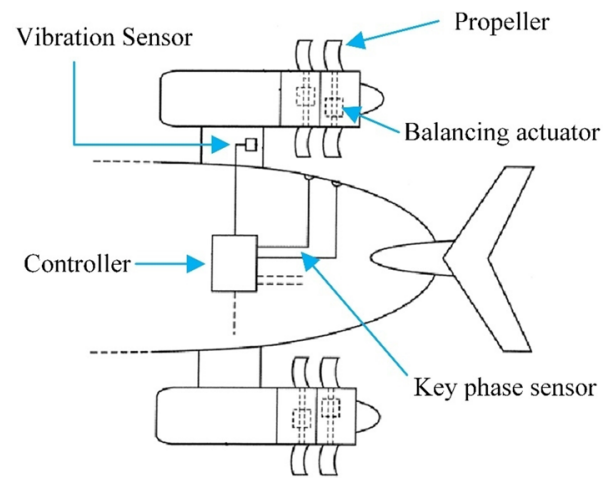

Figure 35 Diagram of actuator for propeller balancing

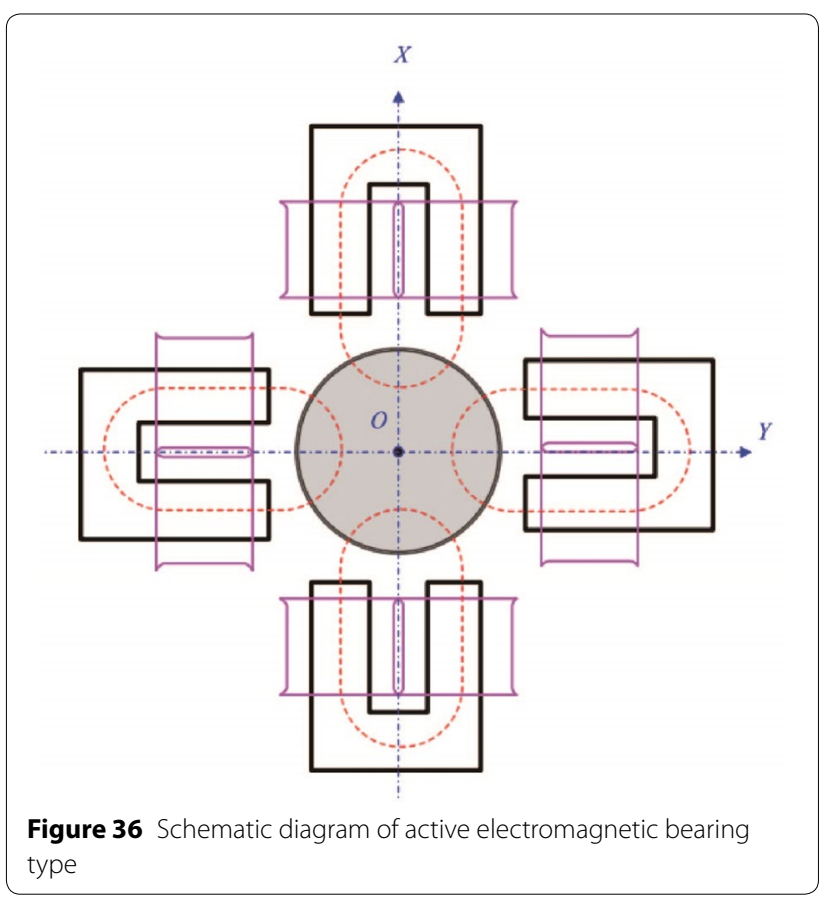

world-leading manufacturing countries have formed the related products, such as USA and Germany [12, 132]. Furthermore, Esteban et al. [133] in Airbus used this type actuator to balance the aircraft propeller, as shown in Figure 35.

\subsubsection{Electromagnetic Actuator}

Electromagnetic balancing devices can be divided into two types: active electromagnetic bearing (AMB) type and electromagnetic ring (EM) type.

For the electromagnetic bearing type, there are multiple sets of coils on the external stator, as shown in Figure 36. When the coils are energized, electromagnetic forces in the corresponding direction will be generated to 
levitate the shaft to support it in the bearing cavity without contact. A synchronous radial force can be applied to the shaft to counterbalance the imbalance [134].

Wang et al. [135] conducted experimental study on a test rig with variable rotating speed, and $A M B$ could minimize unbalance vibration in less than three seconds with non-mistake control strategy. Gao et al. [136] adopted adaptive iterative learning control algorithm as feed-forward compensation to implement real time unbalance vibration compensation. Hutterer et al. [137] described the development of a selfsensing unbalance suppression that an angle sensor is not needed for the unbalance control. Yoon et al. [138] studied the unbalance compensation of the electromagnetic bearing system with input delay, and the method is verified on a test rig. Wan et al. [139] developed a non-contact AMB to suppress the self-excited oscillation of the rotor during milling and improve the surface finish of the machined part, as shown in Figure 37.

For an electromagnetic ring type, the counterweight is driven by the external electromagnetic field generated by the direct current passed in the stator coil, which drives the counterweight disc to a certain position, as shown in Figure 38 [140].

Dyer [141] proposed the first actuator of this kind. Through the alternating magnetic field produced by the static ring, it drives the step rotation of the counterweight in the dynamic ring, and then realizes the online change of the mass distribution. Shin et al. [142] carried out the single-plane and multi-plane automatic balancing experiments of the actuator on the test-bed and the machine tool spindle respectively, which verified the effectiveness of the structure. Moon et al. [76] applied the actuator to high-speed spindle of horizontal machining center, and could reduce spindle unbalance vibration by more than $80 \%$. Su et al. [143] applied this kind of product to high gravity machine. Chen et al. [144] proposed the error free control algorithm to control the actuator, and verified the balance effect in the experimental platform. Fan et al. [145] designed the EM type actuator for

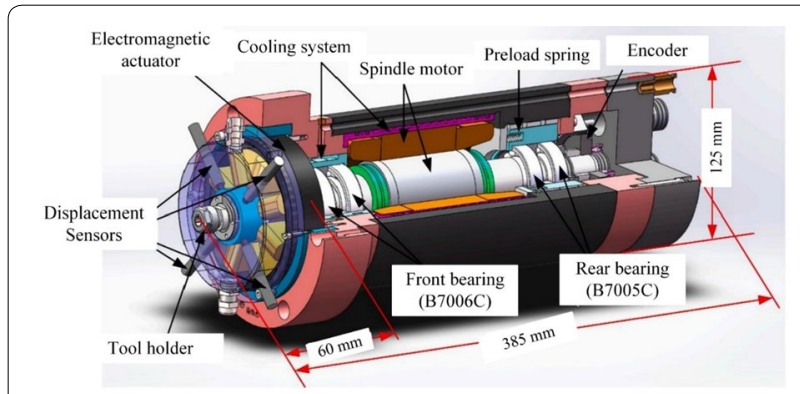

Figure 37 Spindle system with electromagnetic actuator

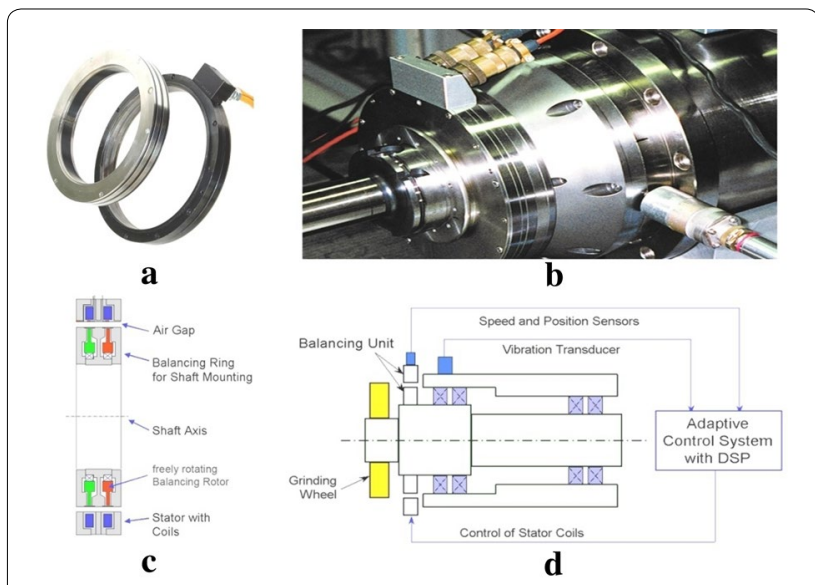

Figure 38 Diagram of electromagnetic ring actuator

the grinding motorized spindle, and the electromagnetic simulations were conducted based on Maxwell software. As mentioned in Section 3.1, this type of actuator has the advantages of fast response speed and high accuracy, and been successfully applied in the balancing of amphibious landing craft and aircraft engine [11].

However, due to the separated structure of coil and counterweight, the existing EM actuator is quite difficult to install on site. To solve the problem, Pan et al. [146] invented an integrated structure of dynamic and static ring, as shown in Figure 39. The equalization experiments were conducted at $3600 \mathrm{r} / \mathrm{min}$, and therefore the novel mechanism can cut back the unbalance vibration by $87.5 \%$ among twenty seconds.

In this chapter, a variety of dynamic balancing actuators that have been commercialized or are in research focus are reviewed. At present, passive actuators have been maturely used in washing machines, hard disk drives and

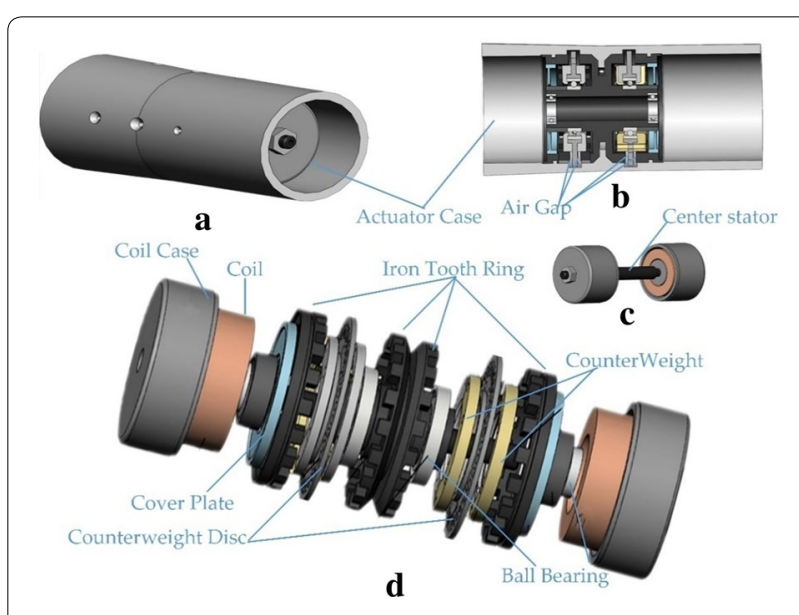

Figure 39 The integrated structure of electromagnetic ring actuator 
other occasions. In addition, active actuators are operated according to the output commands of the controller. Therefore, the balancing process is controllable and has a wider application range. In the future, the simple structure, high precision, and high reliability are the direction of continuous development of these actuators.

\section{Conclusions}

The research on fault self-recovery principle and SR technique has been carried out for nearly two decades. The rapid development of feature extraction, imbalance location, regulation methods and actuator design has promoted the formation of SR technique for unbalance vibration in high-end equipment. The technique includes four levels: condition monitoring, fault diagnosis, targeted control and self-recovery execution. The feasibility and effectiveness of this technique have been verified in the engineering applications in the field of aero-engines and high-end machine tools. On the basis of expounding the basic principle of the technique and related engineering cases, this paper summarizes and analyzes the development of each technology.

Artificial intelligence makes devices smarter and artificial self-recovery makes devices healthier, both of which complement each other. With the popularization of technologies, such as "Intelligent Manufacturing" and "Industry 4.0", the SR technique will develop rapidly at the same time, and greatly reduce the dependence on people for equipment operation and maintenance. It lays a technical foundation for the development of independent and healthy high-end equipment, and has a broad application prospect.

\section{Acknowledgements}

The authors sincerely thanks to National Natural Science Foundation of China (NSFC) for funding and Beijing University of Chemical Technology (BUCT) for providing us with a good platform.

\section{Authors' Contributions}

$\mathrm{XP}$ and JG was in charge of the whole research; $\mathrm{JL}$ and $\mathrm{JH}$ wrote the manuscript; XP and HW modify the manuscript. All authors read and approved the final manuscript.

\section{Authors' Information}

Xin Pan, born in 1987, is currently an associate professor and a master supervisor at Beijing Key Laboratory of Health Monitoring and Self-recovery for High-end Mechanical Equipment, Beijing University of Chemical Technology, China. He received his PhD degree from Beijing University of Chemical Technology, China, in 2015. His research interests include vibration monitoring, fault diagnosis and self-recovery regulation.

Jiaqiao Lu, born in 1995, is currently a master candidate at Beijing Key Laboratory of Health Monitoring and Self-recovery for High-end Mechanical Equipment, Beijing University of Chemical Technology, China.

Jiaji Huo, born in 1996, is currently a master candidate at Beijing Key Laboratory of Health Monitoring and Self-recovery for High-end Mechanical Equipment, Beijing University of Chemical Technology, China.
Jinji Gao, born in 1942, is currently an Academician of Chinese Academy of Engineering and a professor at Key Laboratory of Engine Health Monitoring-Control and Networking (Ministry of Education), Beijing University of Chemical Technology, China. His main research interests include fault diagnosis and self-recovery regulation.

Haiqi Wu, born in 1945, is currently an associate professor at Beijing Key Laboratory of Health Monitoring and Self-recovery for High-end Mechanical Equipment, Beijing University of Chemical Technology, China. His research interests include fault diagnosis and active control.

\section{Funding}

Supported by National Natural Science Foundation of China (Grant No. 51875031) and Youth Backbone Personal Project of Beijing (Grant No. 2017000020124 G018).

\section{Competing Interests}

The authors declare no competing financial interests.

\section{Author Details}

${ }^{1}$ Beijing Key Laboratory of Health Monitoring and Self-recovery for High-end Mechanical Equipment, Beijing University of Chemical Technology, Beijing 100029, China. ${ }^{2}$ Key Laboratory of Engine Health Monitoring-Control and Networking (Ministry of Education), Beijing University of Chemical Technology, Beijing 100029, China.

Received: 25 June 2020 Revised: 11 November 2020 Accepted: 16 November 2020

Published online: 30 November 2020

\section{References}

[1] RY Zhong, XXu, E Klotz, et al. Intelligent manufacturing in the context of Industry 4.0: A review. Engineering, 2017, 3(5): 616-630

[2] HR Cao, XW Zhang, XF Chen. The concept and progress of intelligent spindles: A review. International Journal of Machine Tools and Manufacture, 2017, 112: 21-52

[3] P P Ma, J Y Zhai, H Zhang, et al. Multi-body dynamic simulation and vibration transmission characteristics of dual-rotor system for aeroengine with rubbing coupling faults. Journal of Vibroengineering, 2019, 21(7): 1875-1887.

[4] J Hong, M L Xu, D Y Zhang, et al. Safety design method of fan rotor system in aeroengine due to extreme loadings. Proceeding of the First International Conference on Reliability Systems Engineering ICRSE, Beijing, China, October 21-23, 2015.

[5] PCYu, D Y Zhang, Y H Ma. Dynamic modeling and vibration characteristics analysis of the aero-engine dual-rotor system with Fan blade out. Mechanical Systems And Signal Processing, 2018, 106: 158-175.

[6] Institute for Strategic Research in Advanced Manufacturing. Advanced manufacturing technology in China- a roadmap to 2050. Beijing: Science Press, 2015. (in Chinese)

[7] J J Gao. A study of the fault Self-recovery regulation for process equipment. Proceedings of International Conference on Intelligent Maintenance Systems, Xi'an, China, October 25-27, 2003: 781.

[8] J J Gao. Artificial Self-recovery and machinery self-recovery regulation system. Journal of Mechanical Engineering, 2018, 54(8): 83-94. (in (Chinese)

[9] Consulting Project Group of Chinese Academy of Engineering. Research reports on engineering science and technology and development strategy - Proposal of equipment failure self-recovery engineering and its application in China. Beijing: Chinese Academy of Engineering, 2006. (in (hinese)

[10] J J Gao. Mechanical fault diagnosis and self-recovery. Beijing: Higher Education Press, 2012. (in Chinese)

[11] Corporation LORD. Real-Time Balancing Solutions for the Aerospace \& Defense Industry. 2011 [2020-11-1 1]. https://www.lord.com/china/sites/ china/files/PB6056_PropellerBalancingBrochure.pdf.

[12] Corporation Marposs. Active Control of Vibrations on Grinders. 2005[202011-11]. https://www.marposs.com/chi/download. 
[13] HW Fan, M Q Jing, R C Wang, et al. New electromagnetic ring balancer for active imbalance compensation of rotating machinery. Journal of Sound and Vibration, 2014, 333(17): 3837-3858.

[14] X Pan, H Q Wu, J J Gao. Rotating machinery targeting self-recovery regulation system for imbalance vibration fault with liquid-transfer active balancing Device. Journal of Mechanical Engineering, 2015, 51(1): 146-152.

[15] X L Qiao, C S Zhu. The active unbalanced vibration compensation of the flexible switched reluctance motorized spindle. Journal of Vibration and Control, 2014, 20(13): 1934-1945.

[16] Y S Chen, H B Zhang. Review and prospect on the research of dynamics of complete aero-engine systems. Acta Aeronautica et Astronautica Sinica, 2011, 32(8): 1371-1391.

[17] J J Gao, B Ma, Z N Jiang. Research on fault self-recovery engineering. Journal of Dalian University of Technology, 2006, 46(3): 153-161.

[18] L F Chen, W M Wang, J J Gao. Summary on the development of autobalancing technology of aero-engine. Journal of Aerospace Power, 2019, 34(7): 1530-1541.

[19] E Wilson, K Hale, M Strohmeyer. V-22 rotor track and balance on-board and ground station functionality and field experience. 2011 IEEE Aerospace Conference, AERO 2011, Big Sky, MT, March 5-12, 2011.

[20] W Q Deng, G Tang, D P Gao. Research summary of rotor dynamic characteristics and dynamic balance. Gas Turbine Experiment and Research, 2008, 21(2): 57-62.

[21] BY Chen. Development of aero engine balancing technology. Journal of Propulsion Technology, 1998, 19(4): 7-10.

[22] P Bart, H Wim, D Jan, et al. Modern solutions for ground vibration testing of large aircraft. Sound and Vibration, 2009, 43(1): 8-15.

[23] J L White, M A Heidari, M H Travis. Experience in rotor balancing of large commercial jet engines. 13th International Modal Analysis Conference, Nashville, USA, February 13-19, 1995

[24] B Rebbechi, P R Marsden, S B J Church. C-130J in-flight propeller balancing. In 7th DSTO International Conference on Health \& Usage Monitoring (Australian International Aerospace Congress), 2011.

[25] Q F Xu, SX Lu, WY Jia, et al. Imbalanced fault diagnosis of rotating machinery via multi-domain feature extraction and cost-sensitive learning. Journal of Intelligent Manufacturing, 2019: 1-15.

[26] A S Sait, Y I Sharaf-Eldeen. A review of gearbox condition monitoring based on vibration analysis techniques diagnostics and prognostics. Rotating Machinery, Structural Health Monitoring, Shock and Vibration, 2011, 5: 307-324.

[27] D Goyal, B S Pabla. The vibration monitoring methods and signal processing techniques for structural health monitoring: A review. Archives of Computational Methods in Engineering, 2016, 23(4): 585-594.

[28] A Kumar, R Kumar. Role of signal processing, modeling and decision making in the diagnosis of rolling element bearing defect: A review. Journal of Nondestructive Evaluation, 2019, 38(1): 5.

[29] C Malla, I Panigrahi. Review of condition monitoring of rolling element bearing using vibration analysis and other techniques. Journal of Vibration Engineering \& Technologies, 2019, 7(4): 407-414.

[30] L Deng, R Zhao. Fault feature extraction of a rotor system based on local mean decomposition and Teager energy kurtosis. Journal of Mechanical Science and Technology, 2014, 28(4): 1161-1169.

[31] F D Sanches, R Pederiva. Simultaneous identification of unbalance and shaft bow in a two-disk rotor based on correlation analysis and the SEREP model order reduction method. Journal of Sound \& Vibration, 2018, 433: 230-247.

[32] D He, X F Wang, S Li, et al. Identification of multiple faults in rotating machinery based on minimum entropy deconvolution combined with spectral kurtosis. Mechanical Systems and Signal Processing, 2016, 81: 235-249.

[33] D Ibarra-Zarate, O Tamayo-Pazos, A Vallejo-Guevara. Bearing fault diagnosis in rotating machinery based on cepstrum pre-whitening of vibration and acoustic emission. The International Journal of Advanced Manufacturing Technology, 2019, 104(9-12): 4155-4168.

[34] G Chen. Characteristics analysis of blade-casing rubbing based on casing vibration acceleration. Journal of Mechanical Science and Technology, 2015, 29(4): 1513-1526.

[35] G Chen, Y Q Liu, G Y Jiang, et al. A novel method for identifying rotorstator rubbing positions using the cepstrum analysis technique. Journal of Mechanical Science and Technology, 2014, 28(9): 3537-3544.
[36] H Li, Y P Zhang, H Q Zheng. Gear fault detection and diagnosis under speed-up condition based on order cepstrum and radial basis function neural network. Journal of Mechanical Science and Technology, 2009, 23(10): 2780-2789.

[37] B Liang, S D Iwnicki, Y Zhao. Application of power spectrum, cepstrum, higher order spectrum and neural network analyses for induction motor fault diagnosis. Mechanical Systems and Signal Processing, 2003, 39(1-2): 342-360.

[38] J Urbanek, T Barszcz, R Zimroz, et al. Application of averaged instantaneous power spectrum for diagnostics of machinery operating under non-stationary operational conditions. Measurement, 2012, 45(7): 1782-1791.

[39] I Yesilyurt. Fault detection and location in gears by the smoothed instantaneous power spectrum distribution. NDT and E International, 2003, 36(7): 535-542.

[40] T H Patel, A K Darpe. Application of full spectrum analysis for rotor fault diagnosis. IUTAM Symposium on Emerging Trends in Rotor Dynamics, 2011, 1011: 535-545.

[41] Y G Lei, J Lin, Z J He, et al. A review on empirical mode decomposition in fault diagnosis of rotating machinery. Mechanical Systems and Signal Processing, 2013, 35(1-2): 108-126.

[42] V H Nguyen, J S Cheng, Y Yu, et al. An architecture of deep learning network based on ensemble empirical mode decomposition in precise identification of bearing vibration signal. Journal of Mechanical Science and Technology, 2019, 33(1): 41-50.

[43] HWang, J J Gao, Z N Jiang, et al. Rotating machinery fault diagnosis based on EEMD time-frequency energy and SOM neural network. Arabian Journal for Science and Engineering, 2014, 39(6): 5207-5217.

[44] Z L Xie, B L Shang, RX Zhou. Application of EMD-ICA and demodulation to early failure diagnosis of rotor systems. Proceedings of the First Symposium on Aviation Maintenance and Management, 2014, 296: 361-372.

[45] Y He, D C Pi. Anomaly detection algorithm for helicopter rotor based on STFT and SVDD. Cloud Computing and Security, ICCCS 2016, PT II, 2016, 10040: 383-393.

[46] Y Jin, Z Y Hao, X Zheng. Comparison of different techniques for timefrequency analysis of internal combustion engine vibration signals. Journal of Zhejiang University: Science A, 2011, 12(7): 519-531.

[47] N H Chandra, A S Sekhar. Fault detection in rotor bearing systems using time frequency techniques. Mechanical Systems and Signal Processing, 2016, 72: 105-133

[48] B Li, C L Zhang, Z J He. HHT-based crack identification method for startup rotor. Frontiers of Mechanical Engineering, 2012, 7(3): 300-304.

[49] S M Li, J RWang, X L Li. Theoretical analysis of adaptive harmonic window and its application in frequency extraction of vibration signal. Journal of Central South University, 2018, 25(1): 241-250.

[50] X J Fu. Research on vibration of rotor by application of the improved harmonic wavelet. Analog Integrated Circuits and Signal Processing, 2009, 59(2): 201-205.

[51] V Muralidharan, V Sugumaran. Feature extraction using wavelets and classification through decision tree algorithm for fault diagnosis of mono-block centrifugal pump. Measurement, 2013, 46(1): 353-359.

[52] F Al-Badour, M Sunar, L Cheded. Vibration analysis of rotating machinery using time-frequency analysis and wavelet techniques. Mechanical Systems and Signal Processing, 2011, 25(6): 2083-2101.

[53] Q Hu, Z J He, Z S Zhang, et al. Fault diagnosis of rotating machinery based on improved wavelet package transform and SVMs ensemble. Mechanical Systems and Signal Processing, 2007, 21(2): 688-705.

[54] WT Shan, X A Chen, Y He, et al. A novel experimental research on vibration characteristics of the running high-speed motorized spindles. Journal of Mechanical Science and Technology, 2013, 27(8): 2245-2252.

[55] T H G Lobato, R R da Silva, E S da Costa, et al. An integrated approach to rotating machinery fault diagnosis using, EEMD, SVM, and augmented data. Journal of Vibrational Engineering and Technologies, 2020, 8: 403-408.

[56] X Hu, JVian, J R Slepski, et al. Vibration analysis via neural network inverse models to determine aircraft engine unbalance condition. Neural Networks. International Joint Conference on Neural Networks, Portland, USA, July 20-24, 2003: 3001-3006.

[57] XX Zhu, D N Hou, P Zhou, et al. Rotor fault diagnosis using a convolutional neural network with symmetrized dot pattern images. Measurement, 2019, 138: 526-535. 
[58] J H Yan, Y Y Hu, C Z Guo, Rotor unbalance fault diagnosis using DBN based on multi-source heterogeneous information fusion. Procedia Manufacturing, 2019, 35: 1184-1189.

[59] SY Shao, W J Sun, R Q Yan, et al. A deep learning approach for fault diagnosis of induction motors in manufacturing. Chinese Journal of Mechanical Engineering, 2017, 30(6): 1347-1356.

[60] JT Cheng, L Wang, X Yan. Cuckoo search algorithm with memory and the vibrant fault diagnosis for hydroelectric generating unit. Engineering with Computers, 2019, 35(2): 687-702.

[61] G Li, G L McDonald, Q Zhao. Sinusoidal synthesis based adaptive tracking for rotating machinery fault detection. Mechanical Systems and Signal Processing, 2017, 83: 356-370.

[62] L Lu, J H Yan, CW De Silva. Dominant feature selection for the fault diagnosis of rotary machines using modified genetic algorithm and empirical mode decomposition. Journal of Sound and Vibration, 2015, 344: 464-483.

[63] HX Deng, Y F Diao, W Wu, et al. A high-speed D-CART online fault diagnosis algorithm for rotor systems. Applied Intelligence, 2020, 50(1): 29-41.

[64] Y A Khulief, M A Mohiuddin, M El-Gebeily. A new method for field-balancing of high-speed flexible rotors without trial weights. International Journal of Rotating Machinery, 2014: 603241.

[65] Y A Khulief, W Oke, M A Mohiuddin. Modally tuned influence coefficients for low-speed balancing of flexible rotors. Journal of Vibration and Acoustics, Transactions of the ASME, 2014, 136(2): 024501.

[66] C H Li, Z L Han, C Du, et al. Numerical study on critical speed modeling of ultra-high speed grinder spindle. Advances in Information Technology and Education, 2011, 201: 202-209.

[67] M B Deepthikumar, A S Sekhar, M R Srikanthan. Modal balancing of flexible rotors with bow and distributed unbalance. Journal of Sound and Vibration, 2013, 332(24): 6216-6233.

[68] M S Darlow. Balancing of high-speed machinery: Theory, methods and experimental results. Mechanical Systems and Signal Processing, 1987, 1(1): 105-134.

[69] G F Bin, X J Li, Y P Shen, et al. Development of whole-machine high speed balance approach for turbomachinery shaft system with $\mathrm{N}+1$ supports. Measurement, 2018, 122: 368-379.

[70] Y Kang, TW Lin, Y J Chang, et al. Optimal balancing of flexible rotors by minimizing the condition number of influence coefficients. Mechanism and Machine Theory, 2008, 43(7): 891-908.

[71] TP Goodman. A least-squares method for computing balance corrections. Journal of Engineering for Industry, 1964, 86(3): 273-275.

[72] W P Lei, J Han, H Chen, et al. The modified balance method based on precession decomposition. 2011 IEEE International Conference on Computer Science and Automation Engineering, Shanghai, China, June 10-12, 2011: 632-636.

[73] G Ranjan, R Tiwari. Application of active magnetic bearings for in situ flexible rotor residual balancing using a novel generalized influence coefficient method. Inverse Problems in Science and Engineering, 2019, 27(7): 943-968.

[74] F Fujisawa, K Shiohato, K Sato, et al. Experimental investigation of multispan rotor balancing using least squares method. Journal of Mechanical Design, 1980, 102(3): 589-596.

[75] Y Kang, C P Liu, G J Sheen. A modified influence coefficient method for balancing unsymmetrical rotor-bearing systems. Journal of Sound and Vibration, 1996, 194(2): 199-218.

[76] J D Moon, B S Kim, S H Lee. Development of the active balancing device for high-speed spindle system using influence coefficients. International Journal of Machine Tools and Manufacture, 2006, 46(9): 978-987.

[77] C D Untaroiu, P E Allaire, W C Foiles. Balancing of flexible rotors using convex optimization techniques: Optimum Min-Max LMI influence coefficient balancing. Journal of Vibration and Acoustics, Transactions of the ASME, 2008, 130(2): 021006-021010.

[78] XXu, P P Fan. Rigid rotor dynamic balancing by two-plane correction with the influence coefficient method. Machine Design and Manufacturing Engineering II, PTS 1 and 2, 2013, 365-366: 211-215.

[79] D J Han. Generalized modal balancing for non-isotropic rotor systems. Mechanical Systems and Signal Processing, 2007, 21(5): 2137-2160.

[80] G F Bin, Y Huang, S P Guo, et al. Investigation of induced unbalance magnitude on dynamic characteristics of high-speed turbocharger with floating ring bearings. Chinese Journal of Mechanical Engineering, 2018, 31: 88, https://doi.org/https://doi.org/10.1186/s1003 3-018-0287-5.

[81] C Quartarone, J Beqari, J G Detoni, et al. Balancing of turbomolecular pumps: Modal balancing approach and experimental results. Mechanisms and Machine Science, 2019, 63: 119-128.

[82] G R Wen, X N Zhang, M Zhao. Application of partical swarm optimization algorithm in field holo-balancing. Life System Modeling and Intelligent Computing, 2010, 6328: 16-25.

[83] S Liu. A modified low-speed balancing method for flexible rotors based on holospectrum. Mechanical Systems and Signal Processing, 2006, 21(1): 348-364.

[84] S Liu, L S Qu. A new field balancing method of rotor systems based on holospectrum and genetic algorithm. Applied Soft Computing Journal, 2008, 8(1): 446-455

[85] Y M Ram, J E Mottershead. Multiple-input active vibration control by partial pole placement using the method of receptances. Mechanical Systems and Signal Processing, 2013, 40(2): 727-735.

[86] R Ariyatanapol, Y P Xiong, H J Ouyang. Partial pole assignment with time delays for asymmetric systems. Acta Mechanica, 2018, 229(6): 2619-2629

[87] H Gao, LX Xu. Real-time feed-forward force compensation for active magnetic bearings system based on $\mathrm{H} \infty$ controller. Chinese Journal of Mechanical Engineering, 2011, 24(1): 58-66.

[88] C Liu, D Ye, K Shi, et al. Robust high-precision attitude control for flexible spacecraft with improved mixed $\mathrm{H} 2 / \mathrm{H} \infty$ control strategy under poles assignment constraint. Acta Astronautica, 2017, 136(1): 166-175.

[89] J H Fan, Y M Zhang, Z Q Zheng. Adaptive observer-based integrated fault diagnosis and fault-tolerant control systems against actuator faults and saturation. Journal of Dynamic Systems, Measurement and Control, 2013, 135(4): 0022-0434.

[90] C Peng, J X He, M T Zhu, et al. Optimal synchronous vibration control for magnetically suspended centrifugal compressor, Mechanical Systems and Signal Processing, 2019, 132: 776-789.

[91] G S Tombul, S P Banks. Nonlinear optimal control of rotating flexible shaft in active magnetic bearings. Science China Technological Sciences, 2011, 54(5): 1084-1094.

[92] A Rosyid, M ElMadany, M Alata. Optimal control of reduced-order finite element models of rotor-bearing-support systems. Journal of the Brazilian Society of Mechanical Sciences and Engineering, 2015, 37(5): 1485-1497.

[93] L Zhang, K Liu. Riccati difference equation in optimal control for magnetic bearings. Science China Technological Sciences, 2012, 55(8): 2107-2114.

[94] L H Yang, Y H Sun, L Yu. Active control of unbalance response of rotor systems supported by tilting-pad gas bearings. Proceedings of the Institution of Mechanical Engineers, Part J: Journal of Engineering Tribology, 2012, 226(2): 87-98.

[95] V N Carvalho, B F R Rende, A D G Silva, et al. Robust balancing approach for rotating machines based on fuzzy logic. Journal of Vibration and Acoustics, 2018, 140(5): 051018.

[96] J Xu, Y Zhao, ZY Jia, et al. Rotor dynamic balancing control method based on fuzzy auto-tuning single neuron PID. IEICE Electronics Express, 2017, 14(10): 1349-2543.

[97] W A Oke, M A Abido, T B Asafa. Balancing of flexible rotors based on evolutionary algorithms. Mechanics \& Industry, 2015, 16(4): 406.

[98] X Yang, C Zhang. Optimization for discrete balance weights based on hybrid genetic algorithm. 2011 IEEE International Conference on Computer Science and Automation Engineering, Shanghai, China, June 10-12,2011, 2: 76-79.

[99] D Yao, J Wang, Y Liu. Enhancing working performance of active magnetic bearings using improved fuzzy control and Kalman-LMS filter. Journal of Intelligent \& Fuzzy Systems, 2015, 29(4): 1343-1353.

[100] M L Adams. Rotor balancing and unbalance-caused vibration. Handbook of Noise and Vibration Control, 2008, 57(1): 753-760.

[101] X B Xu, J C Fang, HT Li, et al. Active suppression of imbalance vibration in the magnetically suspended control moment gyro. Journal of Vibration and Control, 2015, 21(5): 989-1003.

[102] S Kwang-Keun. Adaptive control of active balancing systems for speed-varying rotating machinery. University of Michigan, 2001. 
[103] S H Zhang, Z M Zhang, L W Yan. Recent researches and patents on dynamic balancing methods and devices for rotor system. Recent Patents on Mechanical Engineering, 2016, 9(3): 222-229.

[104] E N Pashkov, N V Martyushev, P G Yurovsky. Stationary rotation of the partially liquid-filled unbalanced rotor under external friction force action. High Technology: Research and Applications, 2014, 1040(1): 903-906.

[105] U S Leonardo, L P Marcelo. Liquid self-balancing device effects on flexible rotor stability. Shock and Vibration, 2013, 20(1): 109-121.

[106] D llona, T Viktor, P Kęstutis, et al. Operation of passive fluid self-balancing device at resonance transition regime. Mechanika, 2018, 24(6): 805-810.

[107] P Chao, CW Chiu, KT Shih. A novel low-torque ball re-positioning scheme based on a sliding-mode ball observer for an automatic balancer system. Shock and Vibration, 2008, 15(2): 101-126.

[108] H DeSmidt. Automatic balancing of bladed-disk/shaft system via passive auto-balancer devices. 49th AIAA/ASME/ASCE/AHS/ASC Structures, Structural Dynamics, and Materials Conference 16th AIAA/ASME/AHS Adaptive Structures Conference, Schaumburg, USA, April 7-10, 2008.

[109] V Goncharov, G Filimonikhin, K Dumenko, et al. Studying the peculiarities of balancing of flexible double-support rotors by two passive automatic balancers placed near supports. Eastern-European Journal of Enterprise Technologies, 2016, 4(7): 4-9.

[110] R Shibuya, R Tasaki, KTerashima, Modeling and analysis on stream-tube of pouring flow with a pair of multi-pendulum in self-transfer-type automatic pouring robot. 71st World Foundry Congress: Advanced Sustainable Foundry, Bilbao, Spain, May 18-22, 2014.

[111] YR Su, L D He, W Feng. A study of the active balancing technology for the far end optimization of a cantilever rotor. Journal of Engineering for Thermal Energy and Power, 2008, 23(4): 369-372.

[112] Y R Su, L D He, Z Wang, et al. Study on dual-plane active hydraulic balancing technology for single-disk rigid rotor system. Proceedings of the CSEE, 2009, 29(35): 119-124.

[113] Y Zhang, X S Mei. Study of online balancing control technique for highspeed spindles. Engineering Sciences, 2013, 15(1): 87-92.

[114] X L Yun, X S Mei, G D Jiang, et al. Investigation on a no trial weight spray online dynamic balancer. Shock and Vibration, 2018, 2018: 1-15.

[115] S J Jørren, P Martin. A centrifugal fiberising spinner unit and a method of automatically balancing such unit: DK, 2016008797, 2016-01-21, https://worldwide.espacenet.com/publicationDetails/ originalDocument?FT $=$ D\&date $=20160120 \& D B=E P O D O C \& l o c a l$ $=e n \_E P \& C C=E P \& N R=2975006 A 1 \& K C=A 1 \& N D=4$.

[116] S Z He. Study of liquid release auto-balancing head. Journal of Zhejiang University (Engineering Science), 2001, 35(4): 418-422.

[117] J J Gao, P Zhang. Simulative study of automatic balancing of grinding wheel using a continuously dripping liquid-injection balancing head. WCICA 06, The Sixth World Congress on Intelligent Control and Automation. Piscataway, Dalian, China, June 21-23, 2006: 8002-8005.

[118] Y Li, W M Wang, L Q Huang, et al. A rotor auto-balance device with continuously injecting and draining liquid based on peristaltic pumps. Journal of Vibration and Shock, 2011, 30(4): 38-41.

[119] R Rumin, M Bergander, J Cieslik, et al. Extended active vibration control for wind turbines. 2017 XIIIth International Conference on Perspective Technologies and Methods in MEMS Design (MEMSTECH), Suzhou, China, October 25-27, 2017.

[120] X N Zhang, X Liu, H Zhao. New active online balancing method for grinding wheel using liquid injection and free dripping. Journal of Vibration and Acoustics-Transactions of the ASME, 2018, 140(3): 031001.

[121] J Steere, T Lewandowski. Balanced rotary saw assembly and a method of balancing the same: US, 4537177, 1985-08-27. https://www3.drugf uture.com/uspat/download/US4537177.pdf.

[122] J C Coyne, RT LaGrotta, et al. Liquid-chamber apparatus for active dynamic balancing of rotating machinery: US, 5490436, 1996-02-13. http://www3.drugfuture.com/uspat/download/US5490436.pdf.

[123] K Nakamoto, S Mitsuhashi, H Nakatsuji, et al. Real-time balancing mechanism using magnetic fluid for a machine tool spindle (2nd Report)-Development of a machine tool spindle to verify real-time balancing mechanism. International Journal of the Japan Society of Precision Engineering, 2010, 76(11): 1271-1275.
[124] X N Zhang, X R Xia, Z Xiang, et al. An online active balancing method using magnetorheological effect of magnetic fluid. Journal of Vibration and Acoustics-Transactions of the ASME, 2019, 141(1): 011008.

[125] X Pan, H Q Wu, J J Gao, et al. New liquid transfer active balancing system using compressed air for grinding machine. Journal of Vibration and Acoustics-Transactions of the ASME, 2015, 137(1): 011014.

[126] X Pan, Z Xie, J Lu, et al. Novel liquid transfer active balancing system for hollow rotors of high-speed rotating machinery. Applied Sciences, 2019, 9(5): 833.

[127] Corporation Dittel. Balancing systems for grinding machines. Bologna: Marposs Group, 2012. https://www.dittel.com.

[128] J V Vegte, RT Lake. Balancing of rotating systems during operation. Journal of Sound and Vibration, 1978, 57(2): 225-235.

[129] Y D Kim, CW Lee. Determination of the optimal balancing head location on flexible rotors using a structural dynamics modification algorithm. Proceedings of the Institution of Mechanical Engineers, Part C: Mechanical Engineering Science, 1985, 199(1): 19-25.

[130] Y Li, Y P Lu. Experimental study on vibration control of a PMDF electric balancing head. Journal of Vibration Engineering, 2000, 13(2): 302-306.

[131] B Hredzak, GX Guo. New electromechanical balancing device for active imbalance compensation. Journal of Sound and Vibration, 2006, 294: 737-751.

[132] Corporation Schmitt. Operation and specification manual for the SBS dynamic balance system-Model 4500 series Control Unit. Portland: Accretech SBS Inc, 2020. https://accretechsbs.com/wpcontent/. uploads/MAN-SB-4500-Mechanic-Balancer-EN.pdf.

[133] Q H Esteban. Method for balancing a propulsive system having nonhull contra-rotating propellers: US, 20120328439, 2012-12-27. http:// www3.drugfuture.com/uspat/download/US20120328439.pdf.

[134] Y B Zhang, N Mo, Y Zhou, et al. Unbalance compensation and automatic balance of active magnetic bearing rotor system by using iterative learning control. IEEE Access, 2019, 7: 122613-122625.

[135] W M Wang, J J Gao, L Q Huang, et al. Experimental investigation on vibration control of rotor-bearing system with active magnetic exciter. Chinese Journal of Mechanical Engineering, 2011, 24(6): 1013-1021.

[136] H Gao, LX Xu, Y L Zhu. Unbalance vibratory displacement compensation for active magnetic bearings. Chinese Journal of Mechanical Engineering, 2013, 26(1): 95-103.

[137] M Hutterer, G Kalteis, M Schrodl. Redundant unbalance compensation of an active magnetic bearing system. Mechanical Systems and Signal Processing, 2017, 94: 267-278.

[138] S Y Yoon, L Di, Z L Lin. Unbalance compensation for AMB systems with input delay: An output regulation approach. Control Engineering Practice, 2016, 46: 166-175.

[139] S K Wan, X H Li, W J Su, et al. Active damping of milling chatter vibration via a novel spindle system with an integrated electromagnetic actuator. Precision Engineering, 2019, 57: 203-210.

[140] Company Hofmann. Active Balancing. Pfungstadt: Hofmann Corporation, 2018. https//www.hofmann-balancing.com/products/activebalancing-systems/ring-balancer-ab-9000.html.

[141] SW Dyer, J Ni. Adaptive influence coefficient control of single- plane active balancing systems for rotating machinery. Journal of Manufacturing Science and Engineering, 2001, 123 (3): 291-298.

[142] K K Shin, J Ni. Adaptive control of multi-plane active balancing systems for speed-varying rotors. Journal of Dynamic Systems, Measurement and Control, 2003, 125(9): 372-381.

[143] Y R Su, L D He. Study on online elimination of sudden unbalanceinduced vibration using active balancing technology. High Technology Letters, 2010, 16(2): 209-214.

[144] L F Chen, X Cao, J J Gao. A study on electromagnetic driven bi-disc compensator for rotor auto-balancing and its movement control. WSEAS Transactions on Systems and Control, 2010, 5(5): 333-342.

[145] HW Fan, J J Zhi, B J Shi, et al. Adaptive rotor balancing algorithm and single-disk rotation test for electromagnetic balancer. Journal of Xi'an Jiaotong University, 2018, 52(8): 15-21, 29.

[146] X Pan, TX He, K Z Wei, et al. Performance analysis and experimental research of electromagnetic-ring active balancing actuator for hollow rotors of machine tool spindles. Applied Sciences, 2019, 9(4): 692. 